\title{
EXISTENCE AND MULTIPLICITY OF SOLUTIONS OF AN EQUATION FROM POOL BOILING ON WIRES
}

\author{
BY
}

\author{
SHIN-HWA WANG
}

Department of Mathematics, National Tsing Hua University, Hsinchu, Taiwan 300, Republic of China

\section{Dedicated to Professor Hwai-Chiuan Wang on his 60th birthday}

\begin{abstract}
We investigate the existence and multiplicity of steady states of the equation

$$
\frac{\partial \theta}{\partial t}-\frac{\partial^{2} \theta}{\partial x^{2}}+\sigma q(\theta)-a \lambda(1+\alpha \theta)=0, \quad 0<x<1,
$$
\end{abstract}

with Dirichlet boundary conditions and initial conditions. This equation was derived and studied by Joly, Kernevez, and Llory [7] and Joly [8] in studying thermal effects from pool boiling, in which wires are heated by the Joule effect and are cooled in a bath of boiling water at constant pressure. They studied the steady-state problem for two kinds of heat flux density $q(\theta)$ (corresponding to whether or not the radiation is taken into account) and for $\alpha \neq 0$ or $\alpha=0$. (A) In the case with radiation and $\alpha=0$, for given specific function $q(\theta)$ and constants $\sigma>0, a>0$, by numerical methods, they found an $S$-shaped bifurcation diagram and three solutions for some parameter values. We prove this rigorously, for a specific range of parameters of physical interest. Specifically, we show that, for specific values of $\alpha, \sigma$, and $a$, there exist two positive numbers $\underline{\lambda}<\bar{\lambda}$ such that the steady-state problem has at least three solutions for $\underline{\lambda}<\lambda<\bar{\lambda}$, at least two solutions for $\lambda=\underline{\lambda}$ or $\lambda=\bar{\lambda}$, and exactly one solution for $0 \leq \lambda<\underline{\lambda}$ or $\lambda>\bar{\lambda}$. Moreover, we give lower and upper bounds for $\underline{\lambda}$ and $\bar{\lambda}$. (B) In the case without radiation and $\alpha \neq 0$, we show that there exist two positive numbers $\underline{\lambda}<\bar{\lambda}$ such that the steady-state problem has at least two solutions for $\underline{\lambda}<\lambda<\bar{\lambda}$, at least one solution for $0 \leq \lambda \leq \underline{\lambda}$ or $\lambda=\bar{\lambda}$, exactly one solution for $0<\lambda<\underline{\lambda}$ and $\lambda$ small enough, and no solution for $\lambda>\bar{\lambda}$. Moreover, we give upper and lower bounds for $\underline{\lambda}$ and $\bar{\lambda}$. Also, we find and correct two mistakes in [7, Proposition 2.6, (i), (ii)].

Received May 12, 1998.

2000 Mathematics Subject Classification. Primary 80A20, 34B15, 74G35.

Key words and phrases. Pool boiling, heat transfer, positive solution, bifurcation, S-shaped bifurcation curve.

Work partially supported by Grant NSC 84-2112-M-007-057 from the National Science Council, Republic of China.

E-mail address: shwang@math.nthu.edu.tw 
1. Introduction. We investigate the existence and multiplicity of steady states of the equation

$$
\frac{\partial \theta}{\partial t}-\frac{\partial^{2} \theta}{\partial x^{2}}+\sigma q(\theta)-a \lambda(1+\alpha \theta)=0, \quad 0<x<1
$$

with Dirichlet boundary and initial conditions

$$
\begin{gathered}
\theta(0, t)=\theta(1, t)=0, \\
\theta(x, 0)=\theta_{0}(x) .
\end{gathered}
$$

This reaction-diffusion model equation in (1.1)-(1.3) was derived and studied by Joly, Kernevez, and Llory [7] and Joly [8] in studying thermal effects from pool boiling, in which wires are heated by the Joule effect and are cooled in a bath of boiling water at constant pressure. They assumed that the heat flux density $q$ only depends upon the temperature difference $\theta$ between the heat-transfer temperature and the two-phase flow temperature. In Eq. (1.1), constant $\alpha \geq 0$ is the temperature coefficient of electrical resistance. Positive constants $\sigma, a$ and positive bifurcation parameter $\lambda$ are as follows:

$$
\sigma=\frac{2 l^{2}}{D r}, \quad a=\frac{l^{2} \rho_{0}}{D S^{2}}, \quad \lambda=i^{2}
$$

where $l$ is the wire length, $D$ its thermal conductivity, $r$ its radius, $\rho_{0}$ its specific electrical resistance, $S$ its cross-sectional area, and $i$ is the current intensity along the electric wire $[7]$.

The characieristic boiling curve $q(\theta)$ satisfies that $q(0)=0, q$ increases on an interval $\left(0, \theta_{M}\right)$, then decreases on an interval $\left(\theta_{M}, \theta_{m}\right)$ and last increases for $\theta>\theta_{m}$; see Figs. 1 and 2 . These intervals correspond to three heat-transfer regimes: natural convection and nucleate boiling for $0<\theta<\theta_{M}$, transition boiling for $\theta_{M}<\theta<\theta_{m}$ (in this stage $d q / d \theta<0)$, and film boiling for $\theta>\theta_{m}$ [7, p. 1294]. In particular, the transition boiling region $\left(\theta_{M}, \theta_{m}\right)$ is inherently unstable in experiments in which only the heat flux is controlled, due to the negative slope in the transition region [1, p. 985]. Due to experimental difficulties, the form of the curve representing $q(\theta)$ for $\theta>\theta_{m}$ is not exactly known [7, p. 1294]. Experimentally, the temperature at point $d>\theta_{m}$ with $q(d)=q\left(\theta_{M}\right)$ is in the order of magnitude of the melting point of the metal wires, e.g., the platinum wire. Only when using an extremely thin wire for the heat-transfer surface would the point $(d, q(d))$ really exist $[11$, p. 1421].

Yanagida [15] proposed a simple coupled map lattice model for two-dimensional pool boiling, which exhibits the transition from nucleate to film boiling.

The steady-state equation of (1.1)-(1.3) takes the form

$$
\left\{\begin{array}{l}
-\frac{\partial^{2} \theta}{\partial x^{2}}+\sigma q(\theta)-a \lambda(1+\alpha \theta)=0, \quad 0<x<1 \\
\theta(0)=\theta(1)=0 .
\end{array}\right.
$$

Define

$$
g(\theta)=\sigma q(\theta)-a \lambda(1+\alpha \theta)
$$




$$
G(\theta)=\int_{0}^{\theta} g(u) d u .
$$

Equation (1.5) then can be written as

$$
\left\{\begin{array}{l}
-\frac{\partial^{2} \theta}{\partial x^{2}}+g(\theta)=0, \quad 0<x<1, \\
\theta(0)=\theta(1)=0 .
\end{array}\right.
$$

Clearly, when $\lambda=0$, problem (1.8) has a unique solution $\theta \equiv 0$. Moreover, it is easy to show that, for every $\lambda>0$, the solution $\theta$ of $(1.8)$ is positive; that is,

$$
\theta(x) \geq 0 \text { for every } x \in[0,1]
$$

see [7, Proposition 2.2]. We study the existence and multiplicity of solutions of (1.5) as the bifurcation parameter $\lambda$ varies.

Joly, Kernevez, and Llory [7] and Joly [8] studied (1.5) for the next two cases:

(i) with radiation and $\alpha=0$, as $q(\theta)$ given in Fig. 1 whose data are due to Berenson [1],

(ii) without radiation and with $\alpha \neq 0$, as $q(\theta)$ given in Fig. 2 whose data are due to Madsen [10, Fig. 1].

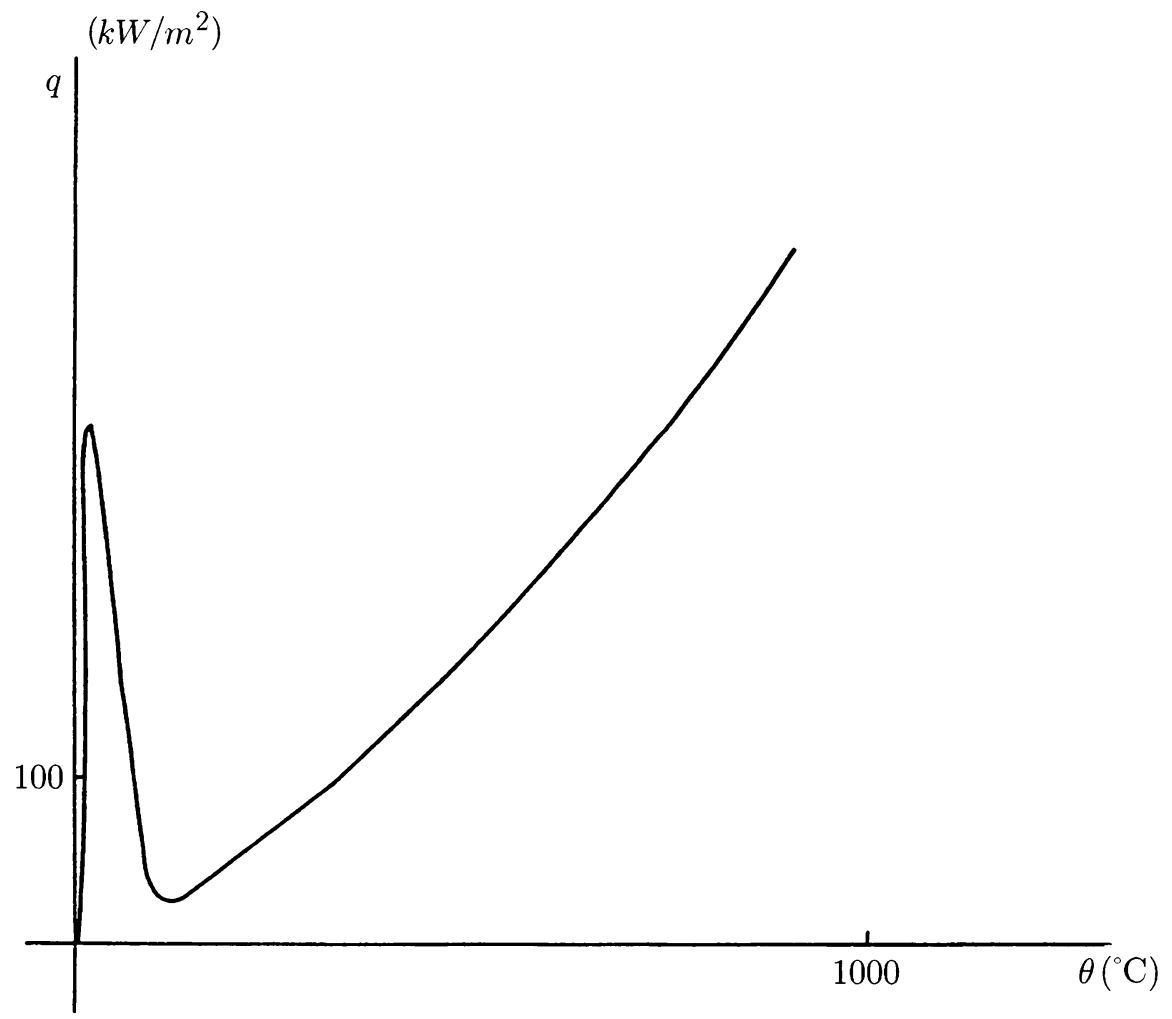

FIG. 1. ([7, Fig. 2] and [8, Fig. 2]). Pool-boiling characteristic in the case with radiation 
They found an $S$-shaped bifurcation diagram and three solutions for (i). While they showed that (1.8) has at least two solutions for a certain range of $\lambda$ and has no solution for $\lambda$ large enough for (ii). However, the work of Joly, Kernevez, and Llory was mainly numerical and, in particular, they only studied particular parameter values, $\alpha=0$, $\sigma=465, a=11560$ for (i), and $\alpha=0.0039, \sigma=172.7, a=1286$ for (ii); see [8, Figs. 1 and 2].

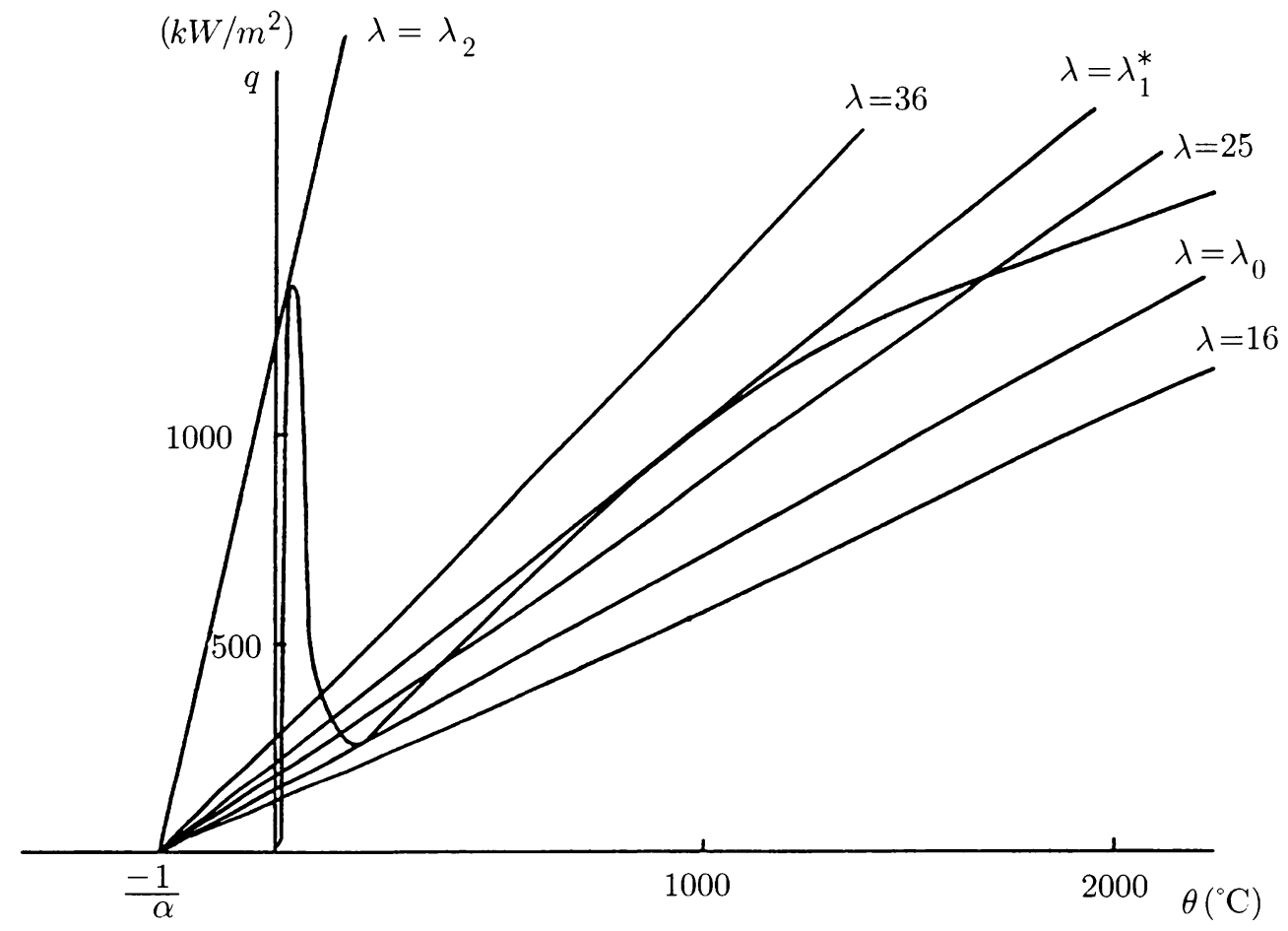

FIG. 2. ([7, Fig. 1] and [8, Fig. 1]). Pool-boiling characteristic in the case without radiation

1.1. Theorems for the case with radiation and $\alpha=0$. In this case radiation is taken into account. The pool-boiling characteristic $q(\theta)$ is given in Fig. 1. The function $q(\theta) \in$ $C^{1}[0, \infty)$ and satisfies

(H1) $q(0)=0$,

(H2) there exist two positive numbers $\theta_{M}<\theta_{m}$ such that

$$
\begin{aligned}
& q^{\prime}(\theta)>0 \text { on }\left(0, \theta_{M}\right), \\
& q^{\prime}(\theta)<0 \text { on }\left(\theta_{M}, \theta_{m}\right), \\
& q^{\prime}(\theta)>0 \text { on }\left(\theta_{m}, \infty\right),
\end{aligned}
$$

(H3) $\lim _{\theta \rightarrow \infty} q(\theta)=\infty$ and $q(\theta)=O\left(\theta^{4}\right)$ as $\theta \rightarrow \infty$ (Boltzmann's fourth-power law). 
We use positive numbers $\lambda_{1}<\lambda_{2}$ as introduced in [7, p. 1300]. $\lambda_{1}$ is chosen such that $g(\theta)=\sigma q(\theta)-a \lambda_{1}=0$ admits three positive roots $\theta_{1}<\theta_{2}<\theta_{3}$ such that

$$
\int_{\theta_{1}}^{\theta_{3}} g(\theta) d \theta=0 .
$$

$\lambda_{2}$ is chosen such that

$$
\sigma q\left(\theta_{M}\right)-a \lambda_{2}=0
$$

Theorem 1.1. Let constants $\alpha=0, \sigma>0, a>0$. Assume that $q(\theta) \in C^{1}[0, \infty)$ satisfies (H1)-(H3). For $\lambda=\lambda_{2}$, suppose that there exist numbers $n, p, r, s, t$, and $m$ satisfying (3.3)-(3.6) stated below, where $f(\theta)$ is defined in (3.4). Then there exist two positive numbers $\underline{\lambda}$ and $\bar{\lambda}$ with $\lambda_{1}<\underline{\lambda}<\lambda_{2}<\bar{\lambda}$ such that problem (1.8) has at least three solutions for $\underline{\lambda}<\lambda<\bar{\lambda}$, at least two solutions for $\lambda=\underline{\lambda}$ or $\lambda=\bar{\lambda}$, and exactly one solution for $0 \leq \lambda<\underline{\lambda}$ or $\lambda>\bar{\lambda}$.

For $q(\theta)$ given in Fig. $1, \alpha=0, \sigma=465$ and $a=11560$, it can be seen that

$$
\begin{aligned}
& 3.3<\lambda_{1}<4.3 \\
& 12<\lambda_{2}<13
\end{aligned}
$$

cf. [7, Figs. 2 and 6].

In the case with radiation and $\alpha=0$, for given specific function $q(\theta)$ and constants $\sigma>0, a>0$, by numerical methods, Joly, Kernevez, and Llory [7] and Joly [8] found an $S$-shaped bifurcation diagram and three solutions for some parameter values; see [7, Proposition 2.3 and section 6]. We prove this rigorously, for a specific range of parameters of physical interest as in the next Theorems 1.2 and 1.3. Theorem 1.2 is an application of Theorem 1.1, in which, with $q(\theta)$ given in Fig. 1, the hypotheses of Theorem 1.1 are verified to hold for parameters $\alpha=0, \sigma=465$, and $a=11560$. Theorem 1.3 is an extension of Theorem 1.2, which is valid for parameters $\alpha=0, \sigma$ and $a$ satisfying (1.15) and (1.16).

TheOREM 1.2. For $q(\theta)$ given in Fig. $1, \alpha=0, \sigma=465, a=11560$, there exist two positive numbers $\underline{\lambda}$ and $\bar{\lambda}$ with $\lambda_{1}<\underline{\lambda}<\lambda_{2}<\bar{\lambda}$ such that problem (1.8) has at least three solutions for $\underline{\lambda}<\lambda<\bar{\lambda}$, at least two solutions for $\lambda=\underline{\lambda}$ or $\lambda=\bar{\lambda}$, and exactly one solution for $0 \leq \lambda<\underline{\lambda}$ or $\lambda>\bar{\lambda}$. Moreover,

$$
\begin{gathered}
3.3<\underline{\lambda}<5.5, \\
12<\bar{\lambda}<13.526 .
\end{gathered}
$$

Hence, for $q(\theta)$ given in Fig. $1, \alpha=0, \sigma=465$ and $a=11560$, problem (1.8) has at least three solutions for $5.5 \leq \lambda \leq 12$.

Theorem 1.2 is consistent with a numerical result obtained by Joly, Kernevez, and Llory [7] in which, for $q(\theta)$ given in Fig. $1, \alpha=0, \sigma=465$ and $a=11560$, they showed numerically that there exist numbers

$$
\underline{\lambda} \approx 3.8, \quad \bar{\lambda} \approx 13.3,
$$


such that problem (1.8) has exactly three solutions for $\underline{\lambda}<\lambda<\bar{\lambda}$; see [7, section 6$]$ for details.

TheOREM 1.3. Let $\underline{\lambda}$ and $\lambda_{2}$ be numbers defined in Theorem 1.2 and (1.10) when $\alpha=0$, $\sigma=465, a=1156$. Let $q(\theta)$ be given in Fig. $1, \alpha=0$, and constants $\sigma$ and $a$ satisfy

$$
\begin{aligned}
\sigma & \geq 465 \\
(11.3943 \approx) \quad \frac{3179}{279} & <\frac{a}{\sigma} \leq \frac{11560}{465}(\approx 24.8602) .
\end{aligned}
$$

Then

(i) problem (1.8) has at least three solutions for $\lambda \in\left(\frac{11560 \sigma}{465 a} \underline{\lambda}, \lambda_{2}\right)$,

(ii) problem (1.8) has exactly one solution for positive $\lambda$ large or small enough.

1.2. Theorems for the case without radiation and with $\alpha \neq 0$. In this case the poolboiling characteristic $q(\theta)$ is given in Fig. 2, it exists for $\theta$ from 0 up to some finite number $\theta_{\infty} \approx 2200$; see [7, Fig. 1] and [10, Fig. 1]. The function $q(\theta) \in C^{1}\left[0, \theta_{\infty}\right)$ and satisfies

(Q1) $q(0)=0$

(Q2) there exist three positive numbers $\theta_{M}<\theta_{m}<\theta_{\infty}(<\infty)$ such that

$$
\begin{aligned}
& q^{\prime}(\theta)>0 \text { on }\left(0, \theta_{M}\right), \\
& q^{\prime}(\theta)<0 \text { on }\left(\theta_{M}, \theta_{m}\right), \\
& q^{\prime}(\theta)>0 \text { on }\left(\theta_{m}, \theta_{\infty}\right),
\end{aligned}
$$

(Q3) there exist exactly three solution-pairs $\left(\lambda_{0}, \theta_{\lambda_{0}}\right),\left(\lambda_{1^{*}}, \theta_{\lambda_{1}^{*}}\right),\left(\lambda_{2}, \theta_{\lambda_{2}}\right)$ of

$$
\left\{\begin{array}{l}
g(\theta)=\sigma q(\theta)-a \lambda(1+\alpha \theta)=0 \\
g^{\prime}(\theta)=\sigma q^{\prime}(\theta)-a \lambda \alpha=0
\end{array}\right.
$$

satisfying

$$
\begin{gathered}
0<\lambda_{0}<\lambda_{1^{*}}<\lambda_{2}, \\
0<\theta_{\lambda_{2}}<\theta_{M}<\theta_{m}<\theta_{\lambda_{0}}<\theta_{\lambda_{1^{*}}}<\theta_{\infty} .
\end{gathered}
$$

First it is obvious to see from Fig. 2 that

$$
\begin{gathered}
16<\lambda_{0}<25 \\
25<\lambda_{1^{*}}<36 \\
140<\lambda_{2}<180 .
\end{gathered}
$$

In particular, (1.19) is obtained by evaluating the $q$ intercepts on the $(\theta, q)$-plane of functions $a \lambda(1+\alpha \theta)$ for $\lambda=36$ and $\lambda=\lambda_{2}$. In this case we look for experimentally meaningful solutions $\theta$ of (1.8) satisfying

$$
0<\|\theta\|_{\infty}<\theta_{\infty}(\approx 2200) \text {. }
$$

Analytically, we may consider (1.8) for an arbitrary function $q(\theta)$ defined for all $\theta \in$ $[0, \infty)$ under additional reasonable asymptotic behavior hypotheses on $q(\theta)$ at infinity. We assume $q(\theta) \in C^{1}[0, \infty)$ and satisfies

(H1) $q(0)=0$, 
(H2) there exist two positive numbers $\theta_{M}<\theta_{m}(<\infty)$ such that

$$
\begin{aligned}
& q^{\prime}(\theta)>0 \text { on }\left(0, \theta_{M}\right), \\
& q^{\prime}(\theta)<0 \text { on }\left(\theta_{M}, \theta_{m}\right), \\
& q^{\prime}(\theta)>0 \text { on }\left(\theta_{m}, \infty\right),
\end{aligned}
$$

$\left(\mathrm{H} 3^{\prime}\right)$ there exist exactly three solution-pairs $\left(\lambda_{0}, \theta_{\lambda_{0}}\right),\left(\lambda_{1^{*}}, \theta_{\lambda_{1^{*}}}\right),\left(\lambda_{2}, \theta_{\lambda_{2}}\right)$ of

$$
\left\{\begin{array}{l}
g(\theta)=\sigma q(\theta)-a \lambda(1+\alpha \theta)=0 \\
g^{\prime}(\theta)=\sigma q^{\prime}(\theta)-a \lambda \alpha=0
\end{array}\right.
$$

satisfying

$$
\begin{gathered}
0<\lambda_{0}<\lambda_{1^{*}}<\lambda_{2}, \\
0<\theta_{\lambda_{2}}<\theta_{M}<\theta_{m}<\theta_{\lambda_{0}}<\theta_{\lambda_{1^{*}}}<\infty,
\end{gathered}
$$

(H4) $\lim _{\theta \rightarrow \infty} \frac{q(\theta)}{\theta}=0, \lim _{\theta \rightarrow \infty}\left(q(\theta)-\theta q^{\prime}(\theta)\right)=\infty$.

Note. Functions $q(\theta)$ behave as $\theta^{\beta}$ with $0<\beta<1$ or as $\theta(\ln \theta)^{-\delta}$ with $\delta>0$ at infinity satisfying (H4).

THEOREM 1.4. Let constants $\alpha>0, \sigma>0$ and $a>0$. Assume that $q(\theta) \in C^{1}[0, \infty)$ satisfies (H1), (H2), (H3'), and (H4). Then there exist two numbers $\underline{\lambda}<\bar{\lambda}$ with

$$
\underline{\lambda}=\frac{\pi^{2}}{a \alpha} \quad \text { and } \quad \lambda_{2}<\bar{\lambda}<\lambda_{2}+\frac{\pi^{2}}{a \alpha}
$$

such that

(i) for $0 \leq \lambda \leq \underline{\lambda}$, problem (1.8) has at least one solution. Moreover, for $0<\lambda<\underline{\lambda}$ and $\lambda$ small enough, problem (1.8) has a unique solution,

(ii) for $\underline{\lambda}<\lambda<\bar{\lambda}$, problem (1.8) has at least two solutions,

(iii) for $\lambda=\bar{\lambda}$, problem (1.8) has at least one solution,

(iv) for $\lambda>\bar{\lambda}$, problem (1.8) has no solution.

It is important to note that, for $\lambda_{0}<\lambda<\lambda_{1^{*}}$,

(A) the equation $g(\theta)=\sigma q(\theta)-a \lambda(1+\alpha \theta)=0$ has four (but not three) positive roots $\theta_{1}<\theta_{2}<\theta_{3}<\theta_{4}\left(\theta_{1}, \theta_{2}, \theta_{3}, \theta_{4}\right.$ depending upon $\left.\lambda\right)$,

(B) there may not exist a number $\lambda=\lambda_{1} \in\left(\lambda_{0}, \lambda_{1^{*}}\right)$ such that

$$
\int_{\theta_{1}}^{\theta_{3}} g(\theta) d \theta=0 \text { for } \quad \lambda=\lambda_{1}
$$

(see $[7$, p. 1302]) since

$$
\int_{\theta_{1}}^{\theta_{3}} g(\theta) d \theta>0 \text { for } \lambda=\lambda_{1^{*}} .
$$

In particular, for function $q(\theta)$ given in Fig. 2 (see also [10, Fig. 1]) and constants $\alpha=0.0039, \sigma=172.7$, and $a=1286,(1.22)$ holds and hence there does not exist a number $\lambda=\lambda_{1}$ with $\lambda_{0}<\lambda_{1}<\lambda_{1^{*}}\left(<\lambda_{2}\right)$ such that (1.21) holds. Thus [7, Proposition 2.6, (i), (ii)] are false. Nevertheless, they can be corrected as in the next theorem.

THEOREM 1.5. Let function $q(\theta)$ be given in Fig. 2 and constants $\alpha=0.0039, \sigma=$ $172.7, a=1286$. Then 
(i) for $0 \leq \lambda \leq 25$, problem (1.8) has a unique solution satisfying (1.20),

(ii) for $40 \leq \lambda \leq 140\left(<\lambda_{2}\right)$, problem (1.8) has at least two solutions satisfying (1.20),

(iii) for $\lambda \geq 182\left(>\lambda_{2}+\left(\pi^{2} /(a \alpha)\right)\right)$, problem (1.8) has no solution satisfying (1.20).

Theorem 1.5 is consistent with a numerical result of Joly, Kernevez, and Llory [7, section 6]; see [7, Fig. 5] in which the solution branch of solutions $\theta$ of problem (1.8) satisfying $0<\|\theta\|_{\infty}<500$ is plotted. They found that problem (1.8) has at least two solutions satisfying $0<\|\theta\|_{\infty}<500$ for $40 \leq \lambda \leq 160$.

We finally remark that it is also of interest to consider problem (1.8) for the next two other cases:

(iii) with radiation and $\alpha \neq 0$ for function $q(\theta)$ satisfying (H1)-(H3) and constants $\alpha>0, \sigma>0, a>0$,

(iv) without radiation and with $\alpha=0$ for function $q(\theta)$ satisfying (H1), (H2), (H3 $\left.{ }^{\prime}\right)$, (H4) and constants $\sigma>0, a>0$.

In either case (iii) or (iv), it can be shown that problem (1.8) has exactly one solution for positive $\lambda$ small or large enough. The exact shape of the solution branch of problem (1.8), similarly as in the two previous cases discussed in Theorems 1.1-1.5, depends significantly upon the function $q(\theta)$ and values of the constants $\alpha \geq 0, \sigma>0, a>0$.

This paper is organized as follows: In Sec. 2, we first give some preliminary lemmas. In Sec. 3, we consider the case with radiation and $\alpha=0$ and prove Theorems 1.1-1.3. Finally, in Sec. 4, we consider the case without radiation and with $\alpha \neq 0$ and prove Theorems 1.4-1.5.

2. Preliminary lemmas. In $[7,8]$, problem (1.8) was studied by analysis of a quadrature formula $k(\gamma)$.

LEmma 2.1. ([7, Lemma 2.2]) There are as many solutions to (1.8) as one can find values of $\gamma$ such that

$$
k(\gamma):=\int_{0}^{\gamma} \frac{d u}{\{2[G(u)-G(\gamma)]\}^{1 / 2}}=\frac{1}{2},
$$

where $G(u)$ is defined in (1.7).

Sometimes, to indicate the dependence more precisely on the bifurcation parameter $\lambda$, we write $g_{\lambda}$ instead of $g, G_{\lambda}$ instead of $G$, and $k_{\lambda}$ instead of $k$ in (1.6)-(1.8) and (2.1), respectively, when necessary. Moreover, to indicate the dependence more precisely on the function $g$, we sometimes write $k^{g}$ (or $k_{\lambda}^{g}$ ) instead of $k$ in (2.1) when necessary.

In order for $k(\gamma)$ to be defined we must have

$$
G(u)>G(\gamma), \quad 0<u<\gamma .
$$

For $\lambda>0$, the set $S$ where $k(\gamma)$ can be defined is generally not an interval. Nevertheless we can show that the set $S \backslash\{0\}$ is an open set; see [12, Proposition 4.1]. Moreover, it is well known that $k \in C^{(n)}$ if $g \in C^{(n)}, n=0,1,2, \ldots$.

The next two lemmas are similar to results in [9], which are useful in studying $k(\gamma)$ and hence the existence and multiplicity of problem (1.8). First, continuous dependence of the time map $k(\gamma)$ on the bifurcation parameter $\lambda$ can be obtained from the representation in (2.1). 
LEMmA 2.2. Suppose that $k_{\lambda}(\gamma)$ exists at $\gamma=\hat{\gamma}>0, \lambda=\hat{\lambda}>0$. Then $k_{\lambda}(\hat{\gamma})$, considered as a function of $\lambda$, is continuous at $\lambda=\hat{\lambda}$.

The next comparison theorem can be shown easily by a generalized mean-value theorem $[4$, p. 118 , Theorem 4]. We omit the proof.

LEMMA 2.3. Suppose that both

$$
k^{g}(\gamma):=\int_{0}^{\gamma} \frac{d u}{\{2[G(u)-G(\gamma)]\}^{1 / 2}}
$$

and

$$
k^{f}(\gamma):=\int_{0}^{\gamma} \frac{d u}{\{2[F(u)-F(\gamma)]\}^{1 / 2}}
$$

exist at $\gamma>0$, where $G(\theta)=\int_{0}^{\theta} g(u) d u$ and $F(\theta)=\int_{0}^{\theta} f(u) d u$. Suppose that $g(\theta) \leq f(\theta)$ for $0<\theta<\gamma$. Then $0<k^{g}(\gamma) \leq k^{f}(\gamma)$. If, additionally, $g(\hat{\theta})<f(\hat{\theta})$ for some $\hat{\theta} \in[0, \gamma)$, then $0<k^{g}(\gamma)<k^{f}(\gamma)$. In particular, let $\alpha \geq 0, \sigma>0, a>0, q(0)=0, q(\theta)>0$ for $\theta>0$. Then

$$
\begin{array}{cc}
g_{\lambda^{*}}(\theta)=\sigma q(\theta)-a \lambda^{*}(1+\alpha \theta), & g_{\lambda^{* *}}(\theta)=\sigma q(\theta)-a \lambda^{* *}(1+\alpha \theta), \\
G_{\lambda^{*}}(\theta)=\int_{0}^{\theta} g_{\lambda^{*}}(u) d u, & G_{\lambda^{* *}}(\theta)=\int_{0}^{\theta} g_{\lambda^{* *}}(u) d u .
\end{array}
$$

Assume that both

$$
k_{\lambda^{*}}(\gamma):=\int_{0}^{\gamma} \frac{d u}{\left\{2\left[G_{\lambda^{*}}(u)-G_{\lambda^{*}}(\gamma)\right]\right\}^{1 / 2}}
$$

and

$$
k_{\lambda^{* *}}(\gamma):=\int_{0}^{\gamma} \frac{d u}{\left\{2\left[G_{\lambda^{* *}}(u)-G_{\lambda^{* *}}(\gamma)\right]\right\}^{1 / 2}}
$$

exist at $\gamma>0$. If $0<\lambda^{*}<\lambda^{* *}$, then $0<k_{\lambda^{* *}}(\gamma)<k_{\lambda^{*}}(\gamma)$.

\section{Proofs of Theorems 1.1-1.3.}

3.1. Proof of Theorem 1.1. First, it is easy to obtain the next lemma. We omit the proof.

Lemma 3.1. Let constants $\sigma>0, a>0$. Let $\lambda \geq 0, g(\theta)=\sigma q(\theta)-a \lambda$, where $q(\theta) \in$ $C^{1}[0, \infty)$ and satisfies (H1)-(H3). Then for any fixed number $\gamma=\hat{\gamma}>0$, there exists a smallest positive number $\lambda=\hat{\lambda}>0$ such that $k_{\lambda}(\hat{\gamma})$ defined by (2.1) exists for $\lambda \in(\hat{\lambda}, \infty)$. Moreover,

$$
\lim _{\lambda \rightarrow \hat{\lambda}^{+}} k_{\lambda}(\hat{\gamma})=\infty \quad \text { and } \quad \lim _{\lambda \rightarrow \infty} k_{\lambda}(\hat{\gamma})=0 .
$$

Let $\lambda \geq 0, g(\theta)=\sigma q(\theta)-a \lambda$, where $q(\theta) \in C^{1}[0, \infty)$ and satisfies (H1)-(H3). Recall the positive number $\lambda_{1}<\lambda_{2}$ introduced in (1.9) and (1.10). $\lambda_{1}$ is chosen such that $g(\theta)=\sigma q(\theta)-a \lambda_{1}=0$ admits three positive roots $\theta_{1}<\theta_{2}<\theta_{3}$ such that $\int_{\theta_{1}}^{\theta_{3}} g(\theta) d \theta=0$. 
$\lambda_{2}$ is chosen such that $\sigma q\left(\theta_{M I}\right)-a \lambda_{2}=0$. For $\lambda_{1}<\lambda<\lambda_{2}, g(\theta)=\sigma q(\theta)-a \lambda=0$ admits three positive roots $\theta_{1}<\theta_{2}<\theta_{3}\left(\theta_{1}, \theta_{2}\right.$, and $\theta_{3}$ depending upon $\left.\lambda\right)$ such that

$$
\int_{\theta_{1}}^{\theta_{3}} g(\theta) d \theta<0 .
$$

So there exists a number $\theta_{5} \in\left(\theta_{2}, \theta_{3}\right)$ such that

$$
\int_{\theta_{1}}^{\theta_{5}} g(\theta) d \theta=0 .
$$

For $\lambda=\lambda_{2}, g(\theta)=\sigma q(\theta)-a \lambda_{2}=0$ admits two positive roots $\theta_{1}<\theta_{2}$. For $\lambda>\lambda_{2}$, $g(\theta)=\sigma q(\theta)-a \lambda=0$ admits one positive root $\theta_{1}$. The next lemma is well known.

Lemma 3.2. (Cf. [13, Fig. 4(b)]) For $\lambda_{1}<\lambda<\lambda_{2}, k_{\lambda}(\gamma)$ exists for every $\gamma \in\left[0, \theta_{1}\right) \cup$ $\left(\theta_{5}, \theta_{3}\right)$. Moreover,

(i) on $\left[0, \theta_{1}\right), k_{\lambda}(\gamma)$ is strictly increasing from 0 to infinity,

(ii) on $\left(\theta_{5}, \theta_{3}\right), \lim _{\gamma \rightarrow \theta_{5}^{+}} k_{\lambda}(\gamma)=\lim _{\gamma \rightarrow \theta_{3}^{-}} k_{\lambda}(\gamma)=\infty$.

For $\lambda=\lambda_{2}, k_{\lambda}(\gamma)$ exists for every $\gamma \in\left[0, \theta_{1}\right) \cup\left(\theta_{1}, \theta_{2}\right)$. Moreover,

(iii) on $\left[0, \theta_{1}\right), k_{\lambda}(\gamma)$ is strictly increasing from 0 to infinity,

(iv) on $\left(\theta_{1}, \theta_{2}\right), \lim _{\gamma \rightarrow \theta_{1}^{+}} k_{\lambda}(\gamma)=\lim _{\gamma \rightarrow \theta_{2}^{-}} k_{\lambda}(\gamma)=\infty$.

For $\lambda>\lambda_{2}, k_{\lambda}(\gamma)$ exists for every $\gamma \in\left[0, \theta_{1}\right)$. Moreover,

(v) on $\left[0, \theta_{1}\right), k_{\lambda}(0)=0, \lim _{\gamma \rightarrow \theta_{1}^{-}} k_{\lambda}(\gamma)=\infty$.

For $\lambda_{1}<\lambda<\lambda_{2}$, by Lemma 3.2, there exists a number $\tilde{\gamma} \in\left(0, \theta_{1}\right)$ such that $k_{\lambda}(\tilde{\gamma})=$ $1 / 2$. For $q(\theta)$ given in Fig. 1 and constants $\alpha=0, \sigma>0$ and $a>0$, suppose that, for some $\lambda=\tilde{\lambda} \in\left(\lambda_{1}, \lambda_{2}\right)$,

$$
\min _{\gamma \in\left(\theta_{5} . \theta_{3}\right)} k_{\tilde{\lambda}}(\gamma)<\frac{1}{2}
$$

see $[7$, p. 1301, lines 27-29]. Then by Lemmas 2.1 and 3.2, problem (1.8) has at least three solutions for $\lambda=\tilde{\lambda}$. Moreover, by Lemmas 2.2 and 2.3, problem (1.8) has at least three solutions for $\tilde{\lambda} \leq \lambda \leq \lambda_{2}$. (Similarly, for $\lambda=\lambda_{2}$, it can be proved that problem (1.8) has at least three solutions.)

For $\lambda=\lambda_{2}$, it is assumed in Theorem 1.1 that there exist numbers $n, p, r, s, t$, and $m$ satisfying

$$
n<0, \quad t<0, \quad 0<p<\theta_{1}<r<s<m<\theta_{2}
$$

such that the curve $S: f(\theta)=0$ which consists of four line segments connecting points $(0, n),(p, 0),(r, 0),(s, t)$ and $(m, 0)$ point by point is above the curve $g_{\lambda_{2}}(\theta)=0$ for $\theta \in[0, m]$ except for $\theta=\theta_{1}$. That is,

$$
\begin{aligned}
& f(\theta):= \begin{cases}\frac{n(p-\theta)}{p} & \text { for } 0 \leq \theta<p, \\
0 & \text { for } p \leq \theta<r, \\
\frac{t(\theta-r)}{(s-r)} & \text { for } r \leq \theta<s, \\
\frac{t(\theta-m)}{(s-m)} & \text { for } s \leq \theta \leq m,\end{cases} \\
& g_{\lambda_{2}}\left(\theta_{1}\right)=f\left(\theta_{1}\right)=0 \text { and } g_{\lambda_{2}}(\theta)=\sigma q(\theta)-a \lambda_{2}<f(\theta) \text { for } \theta \in[0, m] \backslash\left\{\theta_{1}\right\} ;
\end{aligned}
$$

see Fig. 3. 


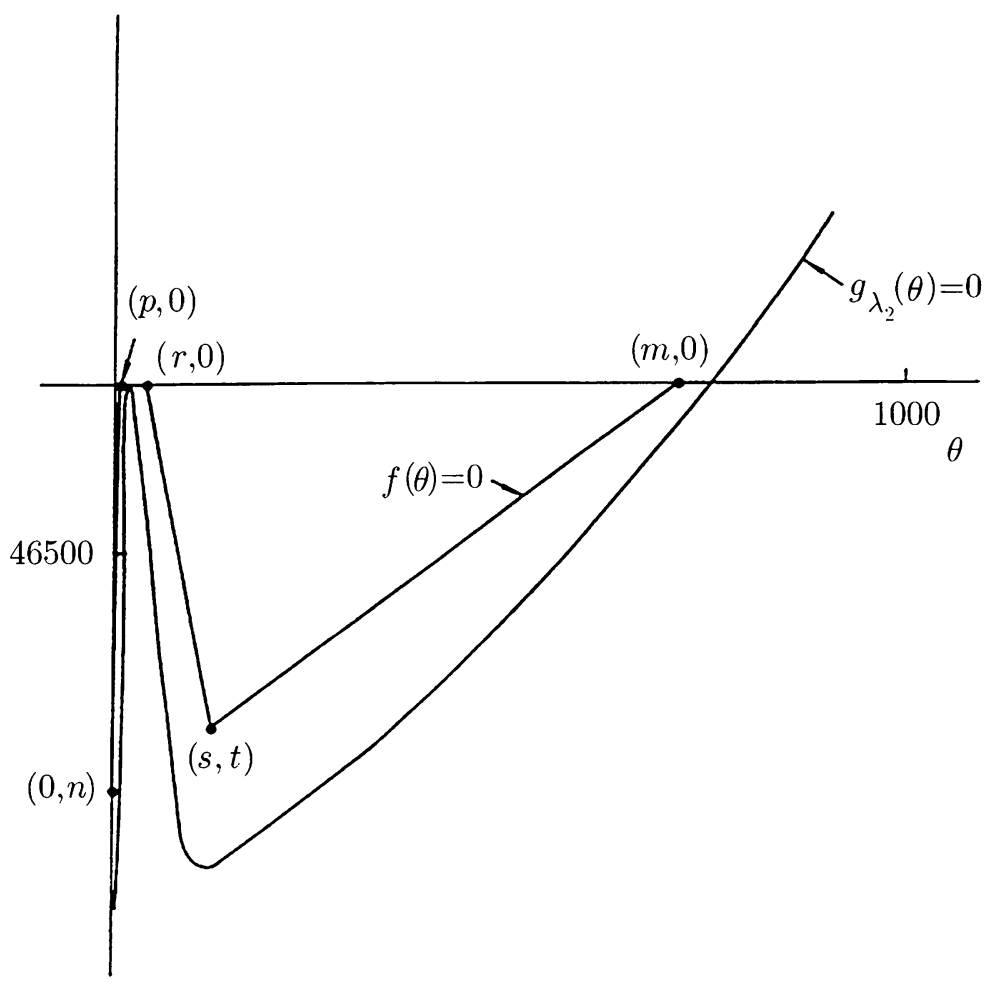

FIG. 3. Curves $f(\theta)=0$ and $g_{\lambda_{2}}(\theta)=0$

\section{Denote}

$$
k_{\lambda_{2}}^{g}(\gamma):=\int_{0}^{\gamma} \frac{d u}{\left\{2\left[G_{\lambda_{2}}(u)-G_{\lambda_{2}}(\gamma)\right]\right\}^{1 / 2}}
$$

and

$$
k^{f}(\gamma):=\int_{0}^{\gamma} \frac{d u}{\{2[F(u)-F(\gamma)]\}^{1 / 2}},
$$

where $G_{\lambda_{2}}(\theta)=\int_{0}^{\theta} g_{\lambda_{2}}(u) d u$ and $F(\theta)=\int_{0}^{\theta} f(u) d u$. It is clear that $k_{\lambda_{2}}^{g}(\gamma)$ and $k^{f}(\gamma)$ exist on $[r, m]$ and on $(r, m)$ respectively. By (3.5) and Lemma 2.3,

$$
0<k_{\lambda_{2}}^{g}(\gamma)<k^{f}(\gamma) \text { for } \gamma \in(r, m) .
$$

It is important to note that $k^{f}(\gamma)$ can be calculated explicitly since $f$ is piecewisely a line segment. (The calculation for $k^{f}(\gamma)$ is straightforward but tedious.) It is assumed in Theorem 1.1 that

$$
\inf _{\gamma \in(r, m)} k^{f}(\gamma)<\frac{1}{2}
$$

Thus

$$
\min _{\gamma \in[r, m]} k_{\lambda_{2}}^{g}(\gamma)<\frac{1}{2}
$$


In these circumstances, let $\lambda=\underline{\lambda} \in\left(\lambda_{1}, \lambda_{2}\right)$ be the number such that

$$
\min _{\gamma \in\left(\theta_{5}, \theta_{3}\right)} k_{\underline{\lambda}}^{g}(\gamma)=\frac{1}{2} \quad\left(\theta_{5} \text { is defined in }(3.1)\right)
$$

for $g_{\underline{\lambda}}(\theta)=\sigma q(\theta)-a \underline{\lambda}$, and let $\bar{\lambda}>\lambda_{2}$ be the largest number such that $k_{\bar{\lambda}}^{g}(\gamma)$ defined on $\left(0, \theta_{1}\right)$ has a local maximum with value $1 / 2$. ( $\underline{\lambda}$ and $\bar{\lambda}$ exist by Lemmas $2.2,2.3,3.1$, and 3.2.) By Lemmas 2.2, 2.3, 3.1, and 3.2, Theorem 1.1 follows.

3.2. Proof of Theorem 1.2. (i) For the function $q(\theta)$ given in Fig. 1, it satisfies (H1)(H3). Let $\alpha=0, \sigma=465, a=11560$, and $\lambda=\lambda_{2}$. We choose

$$
n=-120000, \quad p=10, \quad r=35, \quad s=120, \quad t=-100000, \quad m=720 .
$$

Then (3.3)-(3.5) hold. Moreover, it can be computed that (3.6) holds; see Fig. 4 which is obtained using the symbolic manipulator Mathematica. Thus, by Theorem 1.1, the first part of Theorem 1.2 follows. We next show (1.13) and (1.14) in (ii) and (iii).

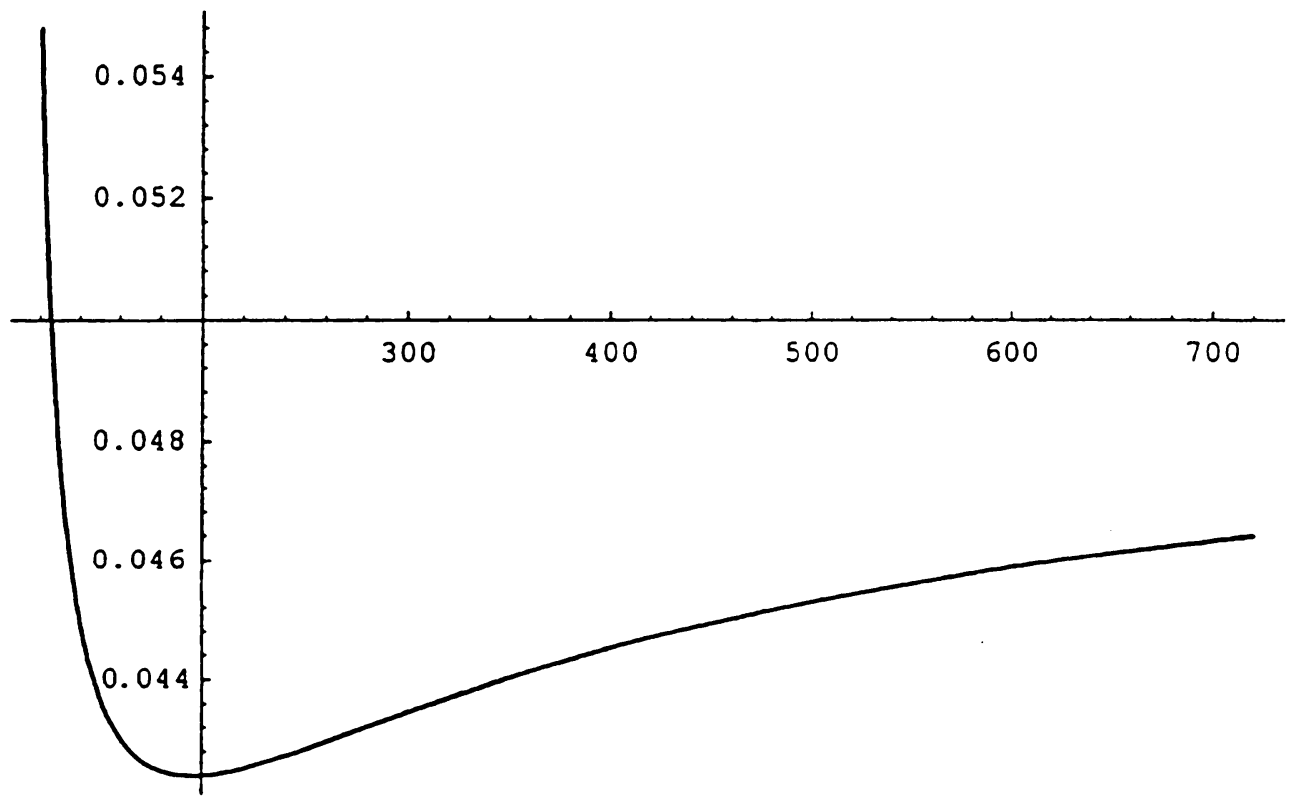

FIG. 4. Curve $k^{f}(\gamma)$ on $(s, m)$

(ii) For $\lambda=\hat{\lambda} \in\left(\lambda_{1}, \lambda_{2}\right), g_{\hat{\lambda}}(\theta)=\sigma q(\theta)-a \hat{\lambda}=0$ admits three positive roots $\theta_{1}<$ $\theta_{2}<\theta_{3}$ such that

$$
\int_{\theta_{1}}^{\theta_{3}} g_{\hat{\lambda}}(\theta) d \theta<0 .
$$

So there exists a number $\theta_{5} \in\left(\theta_{2}, \theta_{3}\right)$ such that

$$
\int_{\theta_{1}}^{\theta_{5}} g_{\hat{\lambda}}(\theta) d \theta=0 .
$$




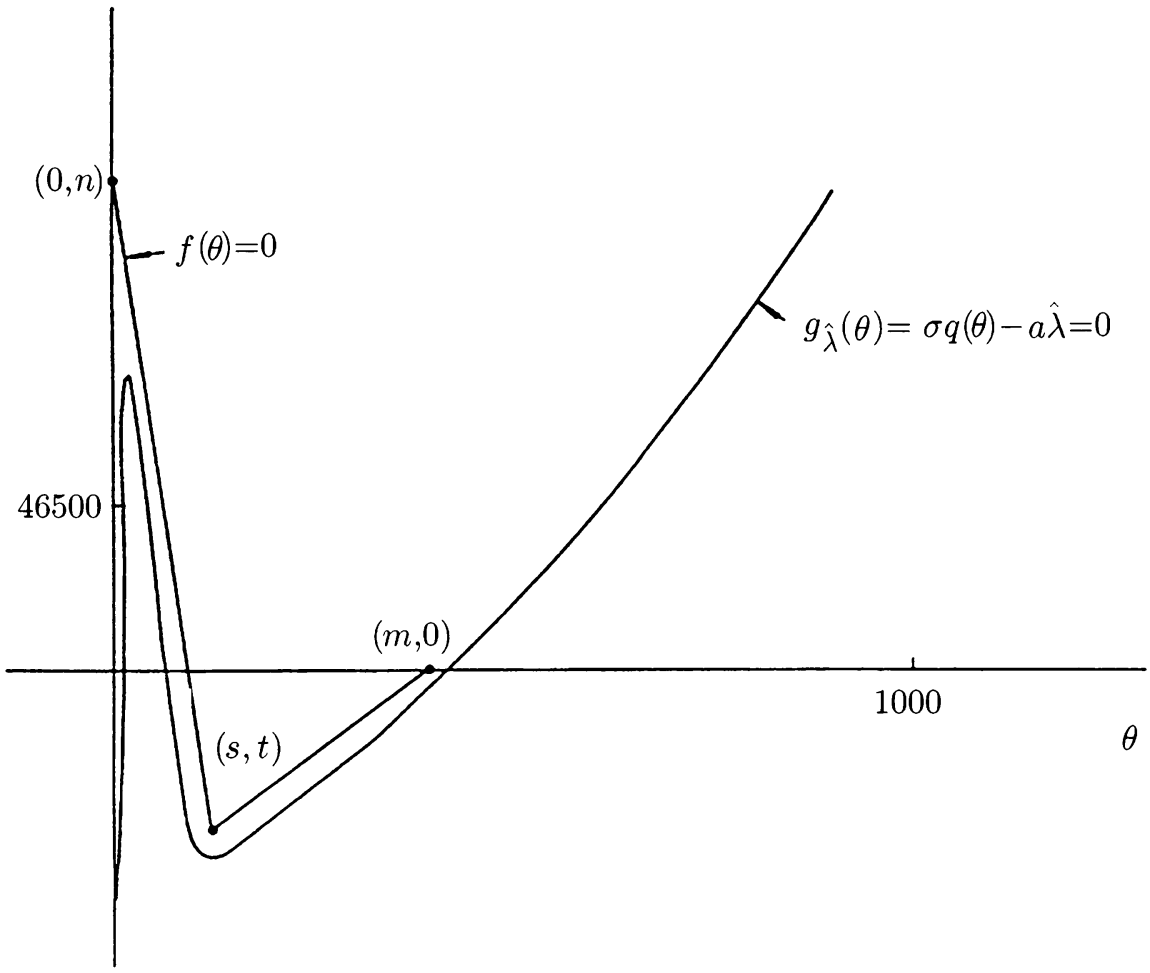

FIG. 5. Curves $f(\theta)=0$ and $g_{\hat{\lambda}}(\theta)=0$

Assume that there exist numbers $n, s, t$, and $m$ satisfying

$$
n>0, \quad t<0, \quad \theta_{2}<s<m<\theta_{3}
$$

such that the curve $V: f(\theta)=0$ which consists of two line segments connecting points $(0, n),(s, t)$ and $(m, 0)$ point by point is above the curve $g_{\hat{\lambda}}(\theta)=0$ on $[0, m]$ for $\lambda=\hat{\lambda}$, i.e.,

$$
g_{\hat{\lambda}}(\theta)=\sigma q(\theta)-a \hat{\lambda}<f(\theta):= \begin{cases}\frac{(t-n) \theta}{s}+n & \text { for } \quad 0 \leq \theta<s \\ \frac{t(\theta-m)}{(s-m)} & \text { for } s \leq \theta \leq m\end{cases}
$$

and $f$ satisfies

$$
\int_{0}^{m} f(\theta) d \theta<0
$$

see Fig. 5. So there exists a number $r \in\left(\theta_{5}, m\right)$ such that

$$
\int_{0}^{r} f(\theta) d \theta=0
$$

Similarly as before, we let

$$
k_{\hat{\lambda}}^{g}(\gamma):=\int_{0}^{\gamma} \frac{d u}{\left\{2\left[G_{\hat{\lambda}}(u)-G_{\hat{\lambda}}(\gamma)\right]\right\}^{1 / 2}}
$$


and

$$
k^{f}(\gamma):=\int_{0}^{\gamma} \frac{d u}{\{2[F(u)-F(\gamma)]\}^{1 / 2}},
$$

where $G_{\hat{\lambda}}(\theta)=\int_{0}^{\theta} g_{\hat{\lambda}}(u) d u$ and $F(\theta)=\int_{0}^{\theta} f(u) d u$. It is clear that $k_{\hat{\lambda}}^{g}(\gamma)$ and $k^{f}(\gamma)$ exist on $[r, m]$ and on $(r, m)$ respectively. By (3.9) and Lemma 2.3,

$$
0<k_{\hat{\lambda}}^{g}(\gamma)<k^{f}(\gamma) \text { for } \gamma \in(r, m) .
$$

$k^{f}(\gamma)$ can be calculated explicitly since $f$ is piecewisely a line segment. (Similarly as before, the calculation for $k^{f}(\gamma)$ is straightforward but tedious.) Hence suppose that

$$
\inf _{\gamma \in(r, m)} k^{f}(\gamma)<\frac{1}{2}
$$

Then

$$
\min _{\gamma \in[r, m]} k_{\hat{\lambda}}^{g}(\gamma)<\frac{1}{2}
$$

for $\lambda=\hat{\lambda} \in\left(\lambda_{1}, \lambda_{2}\right)$. Thus we obtain the next lemma.

Lemma 3.3. For $\lambda=\hat{\lambda} \in\left(\lambda_{1}, \lambda_{2}\right)$, suppose that there exist numbers $n, s$, $t$, and $m$ satisfying (3.8)-(3.12). Then problem (1.8) has at least three solutions for $\hat{\lambda} \leq \lambda \leq \lambda_{2}$. Moreover, let $\underline{\lambda} \in\left(\lambda_{1}, \lambda_{2}\right)$ be defined by (3.7). Then

$$
\left(\lambda_{1}<\right) \underline{\lambda}<\hat{\lambda} \text {. }
$$

For $q(\theta)$ given in Fig. $1, \alpha=0, \sigma=465$, and $a=11560$, we choose

$$
\hat{\lambda}=5.5, \quad n=140000, \quad s=126, \quad t=-44500, \quad m=400 .
$$

Then $r \approx 356.943$ and (3.8)-(3.11) hold. Moreover, it can be computed that

$$
\inf _{\gamma \in(r, m)} k^{f}(\gamma)<0.3<\frac{1}{2}
$$

see Fig. 6 which is obtained using the symbolic manipulator Mathematica. Thus (3.12) holds. So it follows by Lemma 3.3, (1.11) and (3.13) that

$$
3.3<\underline{\lambda}<5.5
$$

and hence (1.13) holds.

(iii) In [7, p. 1301, lines 38-44], for

$$
\lambda>\lambda_{2}+\frac{8 \theta_{p}}{a},
$$

it was shown that (1.8) has a unique solution, where $\theta_{p}>\theta_{m}$ is the number satisfying $a \lambda_{2}=\sigma q\left(\theta_{p}\right)$. For $q(\theta)$ given in Fig. $1, \sigma=465$, and $a=11560$, it is easy to see that

$$
730<\theta_{p}<760 \text {. }
$$

So by Theorem 1.1 and (1.12), we obtain that

$$
12<\lambda_{2}<\bar{\lambda}<\lambda_{2}+\frac{8 \theta_{p}}{a}<13+\frac{8 \cdot 760}{11560}<13.526 .
$$

This proves (1.14). 


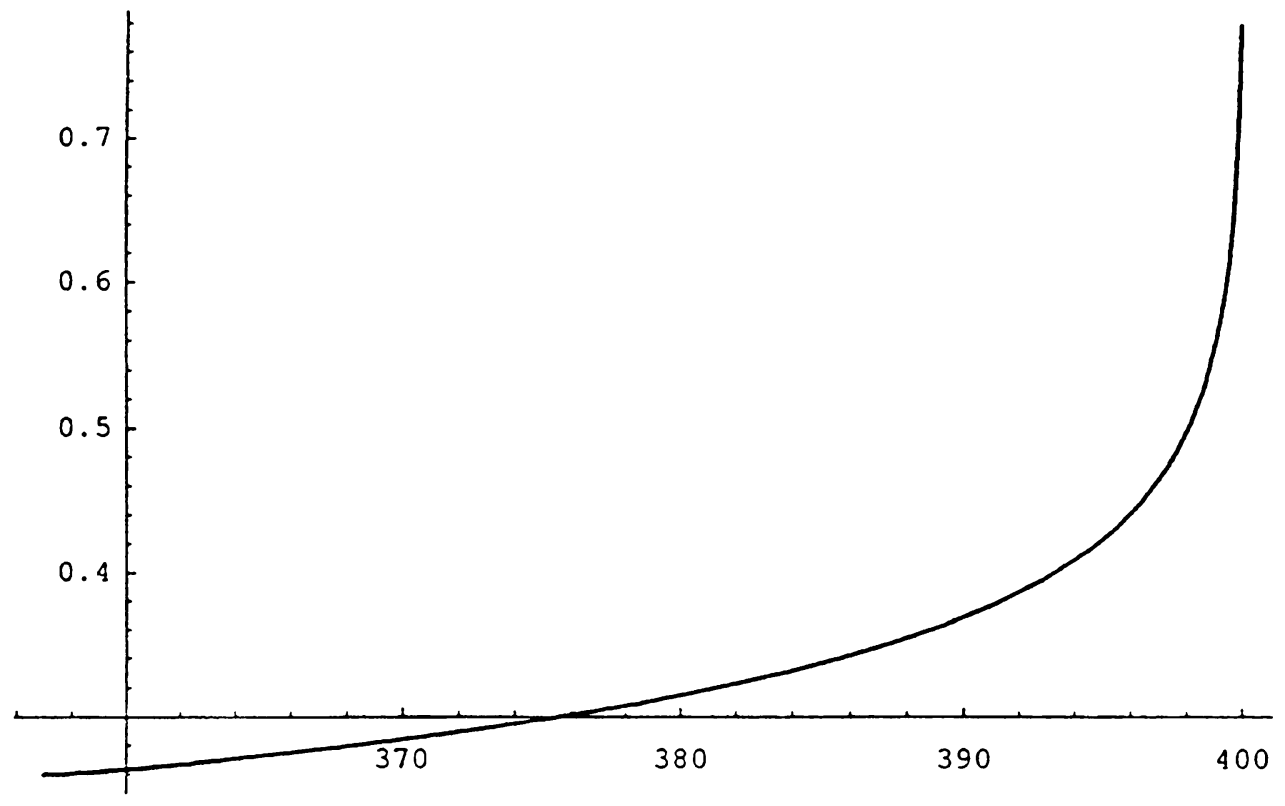

FIG. 6. Curve $k^{f}(\gamma)$ on $(r, m)$

By the above, for $q(\theta)$ given in Fig. $1, \alpha=0, \sigma=465$ and $a=11560$, problem (1.8) has at least three solutions for $5.5 \leq \lambda \leq 12$. The proof of Theorem 1.2 is now complete.

3.3. Proof of Theorem 1.3. Let $\lambda_{1}, \underline{\lambda}$ and $\lambda_{2}$, with $\lambda_{1}<\underline{\lambda}<\lambda_{2}$, be positive numbers defined in (1.9), Theorem 1.2 and (1.10) when $\alpha=0, \sigma=465, a=1156$. Let constants $\sigma=\sigma^{*}$ and $a=a^{*}$ satisfy (1.15) and (1.16). By (1.16), we obtain

$$
1 \leq \frac{11560 \sigma^{*}}{465 a^{*}}
$$

and hence

$$
\underline{\lambda} \leq \frac{11560 \sigma^{*}}{465 a^{*}} \underline{\lambda} .
$$

In addition, by (1.16), (1.12) and (1.13),

$$
\frac{11560 \underline{\lambda}}{465 \lambda_{2}}<\frac{11560 \cdot 5.5}{465 \cdot 12}=\frac{3179}{279}<\frac{a^{*}}{\sigma^{*}}
$$

and hence

$$
\frac{11560 \sigma^{*}}{465 a^{*}} \underline{\lambda}<\lambda_{2}
$$

(3.14) and (3.15) imply that

$$
\underline{\lambda} \leq \frac{11560 \sigma^{*}}{465 a^{*}} \underline{\lambda}<\lambda_{2} .
$$


It follows that

$$
\left(\frac{11560 \sigma^{*}}{465 a^{*}} \underline{\lambda}, \frac{11560 \sigma^{*}}{465 a^{*}} \lambda_{2}\right) \cap\left(\underline{\lambda}, \lambda_{2}\right)=\left(\frac{11560 \sigma^{*}}{465 a^{*}} \underline{\lambda}, \lambda_{2}\right) \neq \emptyset .
$$

By (3.16), for any number $\lambda=\lambda^{*} \in\left(\frac{11560 \sigma^{*}}{465 a^{*}} \underline{\lambda}, \lambda_{2}\right) \subset\left(\underline{\lambda}, \lambda_{2}\right) \subset\left(\lambda_{1}, \lambda_{2}\right)$, we have

$$
\lambda^{*}=\frac{11560 \sigma^{*}}{465 a^{*}} \tilde{\lambda}
$$

for some $\tilde{\lambda} \in\left(\underline{\lambda}, \lambda_{2}\right) \subset\left(\lambda_{1}, \lambda_{2}\right)$. For constants $\sigma>0, a>0, \lambda>0$, define the function

$$
g_{\sigma, a, \lambda}(\theta)=\sigma q(\theta)-a \lambda, \quad \theta \geq 0 .
$$

Then

$$
\begin{aligned}
g_{\sigma^{*}, a^{*}, \lambda^{*}}(\theta) & =\sigma^{*} q(\theta)-a^{*} \lambda^{*} \\
& =\frac{\sigma^{*}}{465}\left(465 q(\theta)-\frac{465 a^{*}}{\sigma^{*}} \lambda^{*}\right) \\
& =\frac{\sigma^{*}}{465}(465 q(\theta)-11560 \tilde{\lambda}) \quad(\text { by }(3.17)) \\
& =\frac{\sigma^{*}}{465} g_{465,11560, \tilde{\lambda}}(\theta) .
\end{aligned}
$$

It is important to note that $g_{\sigma^{*}, a^{*}, \lambda^{*}}(\theta)$ is a constant multiple of $g_{465,11560, \tilde{\lambda}}(\theta)$ and hence functions $g_{\sigma^{*}, a^{*}, \lambda^{*}}(\theta)$ and $g_{465,11560, \tilde{\lambda}}(\theta)$ have the same positive zeros $\theta_{1}<\theta_{2}<\theta_{3}$ and the same value of $\theta_{5}$ defined by (3.1) since $\tilde{\lambda} \in\left(\lambda_{1}, \lambda_{2}\right)$. Moreover, functions $k_{\lambda^{*}}(\gamma, \sigma=$ $\left.\sigma^{*}, a=a^{*}\right)$ and $k_{\tilde{\lambda}}(\gamma, \sigma=465, a=11560)$ defined by (2.1) have the same interval of definition for $\gamma \in\left[0, \theta_{1}\right) \cup\left(\theta_{5}, \theta_{3}\right)$.

By Theorem 1.2 and Lemmas 2.1 and 3.2, for $\lambda=\tilde{\lambda} \in\left(\underline{\lambda}, \lambda_{2}\right) \subset\left(\lambda_{1}, \lambda_{2}\right)$ and $g(\theta)=$ $g_{465,11560, \tilde{\lambda}}(\theta)$,

$$
\min _{\gamma \in\left(\theta_{5}, \theta_{3}\right)} k_{\tilde{\lambda}}(\gamma, \sigma=465, a=11560)=\tilde{t}<\frac{1}{2}
$$

for some positive $\tilde{t}$. By (1.15), (3.18), (3.19) and the formula of $k_{\lambda}$ in (2.1), for $\lambda=\lambda^{*}$ and

$$
g(\theta)=g_{\sigma^{*}, a^{*}, \lambda^{*}}(\theta)=\sigma^{*} q(\theta)-a^{*} \lambda^{*}=\frac{\sigma^{*}}{465} g_{465,11560, \tilde{\lambda}}(\theta)
$$

we obtain that

$$
\begin{aligned}
\min _{\gamma \in\left(\theta_{5}, \theta_{3}\right)} k_{\lambda^{*}}\left(\gamma, \sigma=\sigma^{*}, a=a^{*}\right) & =\left(\frac{\sigma^{*}}{465}\right)^{-1 / 2} \min _{\gamma \in\left(\theta_{5}, \theta_{3}\right)} k_{\tilde{\lambda}}(\gamma, \sigma=465, a=11560) \\
& \leq \min _{\gamma \in\left(\theta_{5}, \theta_{3}\right)} k_{\tilde{\lambda}}(\gamma, \sigma=465, a=11560) \\
& =\tilde{t} \\
& <\frac{1}{2} .
\end{aligned}
$$

Hence, if (1.15) and (1.16) are satisfied, by Lemmas 2.1 and 3.2, problem (1.8) has at least three solutions for $\lambda=\lambda^{*} \in\left(\frac{11560 \sigma^{*}}{465 a^{*}} \underline{\lambda}, \lambda_{2}\right)$. This proves (i). 
The proof of part (ii) of Theorem 1.3 is easy. We omit it. The proof of Theorem 1.3 is now complete.

3.3.1. A remark to Theorems 1.1-1.3. For an arbitrary function $q(\theta)$ satisfying (H1)(H3), $\alpha=0$, and any constants $\sigma>0, a>0$, it is not necessary that problem (1.8) has at least three solutions for some $\lambda \in\left(\lambda_{1}, \lambda_{2}\right)$. This can be shown by the next lemma, which follows easily by the definition of $k(\gamma)$ in $(2.1)$.

LEMmA 3.4. Let the function $q(\theta) \in C^{1}[0, \infty)$ and satisfy (H1)-(H3) and $g(\theta)=\sigma q(\theta)-$ $a \lambda$. Suppose that for $\sigma=\tilde{\sigma}>0, a=\tilde{a}>0$, and $\lambda=\tilde{\lambda}>0, k_{\tilde{\lambda}}(\gamma ; \sigma=\tilde{\sigma}, a=\tilde{a})$ defined by $(2.1)$ exists and

$$
k_{\tilde{\lambda}}(\gamma ; \sigma=\tilde{\sigma}, a=\tilde{a})=t>0 \text {. }
$$

Then for $\sigma=c \tilde{\sigma}, a=c \tilde{a}$, and $\lambda=\tilde{\lambda}, k_{\tilde{\lambda}}(\gamma ; \sigma=c \tilde{\sigma}, a=c \tilde{a})$ exists and

$$
k_{\tilde{\lambda}}(\gamma ; \sigma=c \tilde{\sigma}, a=c \tilde{a})=c^{-1 / 2} k_{\tilde{\lambda}}(\gamma ; \sigma=\tilde{\sigma}, a=\tilde{a})=c^{-1 / 2} t
$$

for any constant $c>0$.

In Lemma 3.4, functions $\tilde{\sigma} q(\theta)-\tilde{a} \lambda$ and $(c \tilde{\sigma}) q(\theta)-(c \tilde{a}) \lambda$ have the same zeros and the same values of $\lambda_{1}$ and $\lambda_{2}$. In particular, they have the same zeros (i) $\theta_{1}<\theta_{2}<\theta_{3}$ for $\lambda_{1}<\lambda<\lambda_{2}$ and (ii) $\theta_{1}<\theta_{2}$ for $\lambda=\lambda_{2}$. Define constants $\sigma=\tilde{\sigma}>0, a=\tilde{a}>0$. Suppose that, for some $\lambda=\tilde{\lambda} \in\left(\lambda_{1}, \lambda_{2}\right), k_{\tilde{\lambda}}(\gamma ; \sigma=\tilde{\sigma}, a=\tilde{a})$ has its minimum on $\left(\theta_{5}, \theta_{3}\right)$ at $\gamma=\tilde{\gamma}>0$, and

$$
k_{\tilde{\lambda}}(\tilde{\gamma} ; \sigma=\tilde{\sigma}, a=\tilde{a})=\tilde{t} \leq \frac{1}{2} \quad(\tilde{t}>0)
$$

see Lemma 3.2. Then $k_{\tilde{\lambda}}(\gamma ; \sigma=c \tilde{\sigma}, a=c \tilde{a})$ has the same interval of definition as that of $k_{\tilde{\lambda}}(\gamma ; \sigma=\tilde{\sigma}, a=\tilde{a})$. Moreover, $k_{\tilde{\lambda}}(\gamma ; \sigma=c \tilde{\sigma}, a=c \tilde{a})$ has its minimum on $\left(\theta_{5}, \theta_{3}\right)$ at $\gamma=\tilde{\gamma}$, and

$$
k_{\tilde{\lambda}}(\tilde{\gamma} ; \sigma=c \tilde{\sigma}, a=c \tilde{a})=c^{-1 / 2} k_{\tilde{\lambda}}(\tilde{\gamma} ; \sigma=\tilde{\sigma}, a=\tilde{a})=c^{-1 / 2} \tilde{t}>\frac{1}{2}
$$

if the positive number $c$ is small enough. (3.20)-(3.21) and Lemmas 2.2-2.3 imply: In order that the results of Theorems 1.1-1.3 hold, in addition to the given function $q(\theta)$, it significantly depends upon the values of $\sigma$ and $a$. In the case that, for an arbitrary function $q(\theta)$ satisfying (H1)-(H3) and any constants $\sigma>0, a>0$,

$$
k_{\lambda_{2}}(\gamma)>\frac{1}{2} \text { for any } \gamma \in\left(\theta_{1}, \theta_{2}\right) \text {, }
$$

and thus problem (1.8) has exactly one solution for $0 \leq \lambda \leq \lambda_{2}$. It is still possible that, for some $\lambda=\hat{\lambda}>\lambda_{2}$, the equality

$$
k_{\hat{\lambda}}(\gamma)=\frac{1}{2}
$$

has at least three solutions for $\gamma>0$, and hence problem (1.8) has at least three solutions. In this case, to study the global bifurcation diagram for problem (1.8) on the $\left(\lambda,\|\theta\|_{\infty}\right)$ plane, further understanding on the quadrature formula $k_{\lambda}(\gamma)$ for $\lambda>\lambda_{2}$ is necessary. 


\section{Proofs of Theorems 1.4-1.5.}

4.1. Proof of Theorem 1.4. In Theorem 1.4 we look for solutions $\theta$ of (1.8) satisfying

$$
0<\|\theta\|_{\infty}<\infty
$$

For any given function $q(\theta)$ satisfying $(\mathrm{H} 1),(\mathrm{H} 2),\left(\mathrm{H}^{\prime}\right)$, and (H4) and any given positive constants $\sigma, a, \alpha$, the equation

$$
g(\theta)=\sigma q(\theta)-a \lambda(1+\alpha \theta)=0 \quad(\lambda>0)
$$

has at most 4 positive roots $\theta_{1} \leq \theta_{2} \leq \theta_{3} \leq \theta_{4}$ which can be the same. More precisely,

(i) for $0<\lambda<\lambda_{0}, g(\theta)=0$ has two distinct positive roots $\theta_{1}<\theta_{4}$,

(ii) for $\lambda=\lambda_{0}, g(\theta)=0$ has three distinct positive roots $\theta_{1}<\theta_{2}<\theta_{4}$,

(iii) for $\lambda_{0}<\lambda<\lambda_{1^{*}}, g(\theta)=0$ has four distinct positive roots $\theta_{1}<\theta_{2}<\theta_{3}<\theta_{4}$,

(iv) for $\lambda=\lambda_{1^{*}}, g(\theta)=0$ has three distinct positive roots $\theta_{1}<\theta_{2}<\theta_{3}$,

(v) for $\lambda_{1^{*}}<\lambda<\lambda_{2}, g(\theta)=0$ has two distinct positive roots $\theta_{1}<\theta_{2}$,

(vi) for $\lambda=\lambda_{2}, g(\theta)=0$ has one positive $\operatorname{root} \theta_{1}$,

(vii) for $\lambda>\lambda_{2}, g(\theta)=0$ has no positive root,

cf. Fig. 2. By the above,

(i) for $0<\lambda<\lambda_{0}$, there exists a number $\theta_{5}>\theta_{4}$ satisfying $\int_{\theta_{1}}^{\theta_{5}} g(\theta) d \theta=0$ such that $k(\gamma)$ exists for $\gamma \in\left[0, \theta_{1}\right) \cup\left(\theta_{5}, \infty\right)$,

(ii) for $\lambda=\lambda_{0}$, there exists a number $\theta_{5}>\theta_{4}$ satisfying $\int_{\theta_{1}}^{\theta_{5}} g(\theta) d \theta=0$ such that $k(\gamma)$ exists for $\gamma \in\left[0, \theta_{1}\right) \cup\left(\theta_{5}, \infty\right)$,

(iii) for $\lambda_{0}<\lambda<\lambda_{1^{*}}$, if $\int_{\theta_{1}}^{\theta_{3}} g(\theta) d \theta \geq 0$, then there exists a number $\theta_{5}>\theta_{4}$ satisfying $\int_{\theta_{1}}^{\theta_{5}} g(\theta) d \theta=0$ such that $k(\gamma)$ exists for $\gamma \in\left[0, \theta_{1}\right) \cup\left(\theta_{5}, \infty\right)$; if $\int_{\theta_{1}}^{\theta_{3}} g(\theta) d \theta<0$, then there exist two numbers $\theta_{5}, \theta_{6}$ with $\theta_{2}<\theta_{6}<\theta_{3}<\theta_{4}<\theta_{5}$ satisfying $\int_{\theta_{1}}^{\theta_{6}} g(\theta) d \theta=$ 0 and $\int_{\theta_{3}}^{\theta_{5}} g(\theta) d \theta=0$ such that $k(\gamma)$ exists for $\gamma \in\left[0, \theta_{1}\right) \cup\left(\theta_{6}, \theta_{3}\right) \cup\left(\theta_{5}, \infty\right)$,

(iv) for $\lambda=\lambda_{1^{*}}$, if $\int_{\theta_{1}}^{\theta_{3}} g(\theta) d \theta \geq 0$, then there exists a number $\theta_{5}>\theta_{3}$ satisfying $\int_{\theta_{1}}^{\theta_{5}} g(\theta) d \theta=0$ such that $k(\gamma)$ exists for $\gamma \in\left[0, \theta_{1}\right) \cup\left(\theta_{5}, \infty\right)$; if $\int_{\theta_{1}}^{\theta_{3}} g(\theta) d \theta<0$, then there exist two numbers $\theta_{5}, \theta_{6}$ with $\theta_{2}<\theta_{6}<\theta_{3}<\theta_{5}$ satisfying $\int_{\theta_{1}}^{\theta_{6}} g(\theta) d \theta=0$ and $\int_{\theta_{3}}^{\theta_{5}} g(\theta) d \theta=0$ such that $k(\gamma)$ exists for $\gamma \in\left[0, \theta_{1}\right) \cup\left(\theta_{6}, \theta_{3}\right) \cup\left(\theta_{5}, \infty\right)$,

(v) for $\lambda_{1^{*}}<\lambda<\lambda_{2}$, there exists a number $\theta_{5}>\theta_{2}$ satisfying $\int_{\theta_{1}}^{\theta_{5}} g(\theta) d \theta=0$ such that $k(\gamma)$ exists for $\gamma \in\left[0, \theta_{1}\right) \cup\left(\theta_{5}, \infty\right)$,

(vi) for $\lambda=\lambda_{2}, k(\gamma)$ exists for $\gamma \in\left[0, \theta_{1}\right) \cup\left(\theta_{1}, \infty\right)$,

(vii) for $\lambda>\lambda_{2}, k(\gamma)$ exists for $\gamma \in[0, \infty)$.

First, it is easy to obtain the next two lemmas. We omit the proofs.

Lemma 4.1. (cf. Lemma 3.1) Let constants $\alpha>0, \sigma>0$ and $a>0$. Let $g(\theta)=$ $\sigma q(\theta)-a \lambda(1+\alpha \theta)$, where $q(\theta) \in C^{1}[0, \infty)$ satisfies (H1), (H2), (H3') and (H4). Then, for any fixed number $\gamma=\hat{\gamma}>0$, there exists a smallest positive number $\lambda=\hat{\lambda}>0$ such 
that $k_{\lambda}(\hat{\gamma})$ defined by $(2.1)$ exists for $\lambda \in(\hat{\lambda}, \infty)$. Moreover,

$$
\lim _{\lambda \rightarrow \hat{\lambda}^{+}} k_{\lambda}(\hat{\gamma})=\infty \quad \text { and } \quad \lim _{\lambda \rightarrow \infty} k_{\lambda}(\hat{\gamma})=0
$$

Lemma 4.2. (cf. [7, p. 1301, Lemma 2.4]) Let constants $\alpha>0, \sigma>0$ and $a>0$. Let $g(\theta)=\sigma q(\theta)-a \lambda(1+\alpha \theta)$, where $q(\theta) \in C^{1}[0, \infty)$ satisfies (H1), (H2), (H3') and (H4). Then, for $0<\lambda \leq \lambda_{2}$,

(i) $k(\gamma)$ exists for every $\gamma \in\left[0, \theta_{1}\right), k(0)=0$ and $\lim _{\gamma \rightarrow \theta_{1}^{-}} k(\gamma)=\infty$,

(ii) the derivative $k^{\prime}(\gamma)$ exists and $k^{\prime}(\gamma)>0$ on $\left(0, \theta_{1}\right)$.

Moreover, we have

Lemma 4.3. Let constants $\alpha>0, \sigma>0$ and $a>0$. Let $\lambda>0, g(\theta)=\sigma q(\theta)-a \lambda(1+\alpha \theta)$, where $q(\theta) \in C^{1}[0, \infty)$ satisfies $(\mathrm{H} 1),(\mathrm{H} 2),\left(\mathrm{H}^{\prime}\right)$ and $(\mathrm{H} 4)$. Then

(i) $\lim _{\gamma \rightarrow \infty} k(\gamma)=(\pi / 2)(a \lambda \alpha)^{-1 / 2}$,

(ii) $k^{\prime}(\gamma)<0$ if $\gamma$ is large enough.

Proof. (i) Suppose that $q(\theta)$ satisfies (H1), (H2), (H3') and (H4). Then

$$
\lim _{\theta \rightarrow \infty} \frac{q(\theta)}{\theta}=a \lambda \alpha \text {. }
$$

Hence, it is well known that

$$
\lim _{\gamma \rightarrow \infty} k(\gamma)=(\pi / 2)(a \lambda \alpha)^{-1 / 2}
$$

see $[9$, Theorem 3.1].

(ii) It can be calculated that

$$
k^{\prime}(\gamma)=\int_{0}^{\gamma} \frac{H(u)-H(\gamma)}{\{2[G(u)-G(\gamma)]\}^{3 / 2}} \frac{d u}{\gamma},
$$

where

$$
\begin{aligned}
H(x) & =2 G(x)-x g(x) \\
& =2 \sigma Q(x)-a \lambda\left(2 x+\alpha x^{2}\right)-x[\sigma q(x)-a \lambda(1+\alpha x)] \quad\left(Q(x):=\int_{0}^{x} q(s) d s\right) \\
& =\sigma[2 Q(x)-x q(x)]-a \lambda x ;
\end{aligned}
$$

see, e.g., $[13,(1.8)]$. Hence by (H4),

$$
\lim _{x \rightarrow \infty} H^{\prime}(x)=\lim _{x \rightarrow \infty}\left\{\sigma\left[q(x)-x q^{\prime}(x)\right]-a \lambda\right\}=\sigma \lim _{x \rightarrow \infty}\left[q(x)-x q^{\prime}(x)\right]-a \lambda=\infty .
$$

Thus for $k^{\prime}(\gamma)$ in (4.3), it is easy to see that $k^{\prime}(\gamma)<0$ if $\gamma$ is large enough. This completes the proof of Lemma 4.3 .

Lemma 4.4. Let constants $\alpha>0, \sigma>0$ and $a>0$. Let $\lambda>0, g(\theta)=\sigma q(\theta)-a \lambda(1+\alpha \theta)$, where $q(\theta) \in C^{1}[0, \infty)$ satisfies (H1), (H2), (H3') and (H4).

(i) For $0<\lambda \leq \lambda_{2}$, problem (1.8) has at least one solution. Moreover, for $0<\lambda<\lambda_{2}$ and $\lambda$ small enough, problem (1.8) has a unique solution.

(ii) For $\lambda>\lambda_{2}+\left(\pi^{2} /(a \alpha)\right)$, problem (1.8) has no solution. 
Lemma 4.4, (ii) improves [7, Proposition 2.6, (iii)] slightly.

Proof. Let

$$
k^{g}(\gamma):=\int_{0}^{\gamma} \frac{d u}{\{2[G(u)-G(\gamma)]\}^{1 / 2}} \quad\left(G(\theta)=\int_{0}^{\theta} g(u) d u\right) .
$$

(i) For $0<\lambda \leq \lambda_{2}$, by Lemma 4.2, problem (1.8) has at least one solution satisfying (4.1). Moreover, for $0<\lambda<\lambda_{0}, g(\theta)=\sigma q(\theta)-a \lambda(1+\alpha \theta)=0$ has two positive roots $\theta_{1}<\theta_{4}$, and there exists a number $\theta_{5}>\theta_{4}$ such that

$$
\int_{\theta_{1}}^{\theta_{5}} g(u) d u=0
$$

$\theta_{5}$ depends on $\lambda$ and is a strictly decreasing function of $\lambda<\lambda_{0}$. Furthermore,

$$
\lim _{\lambda \rightarrow 0} \theta_{5}=\infty \text {. }
$$

For $0<\lambda \leq \lambda_{2}, k^{g}(\gamma)$ exists for $\gamma \in\left[0, \theta_{1}\right) \cup\left(\theta_{5}, \infty\right)$. It is known in Lemma 4.2 that
(A) $k^{g}(0)=0$,
(B) $\lim _{\gamma \rightarrow \theta_{1}^{-}} k^{g}(\gamma)=\infty$,
(C) $\left(k^{g}\right)^{\prime}(\gamma)>0$ on $\left(0, \theta_{1}\right)$.

In addition, for $0<\lambda \leq \pi^{2} /(2 a \alpha)$,

$$
g(\theta)=\sigma q(\theta)-a \lambda(1+\alpha \theta)>-a \lambda(1+\alpha \theta):=f(\theta) \text { for } \theta>0 .
$$

By Lemma 2.3,

$$
k^{g}(\gamma)>k^{f}(\gamma)>0 .
$$

For $f(\theta)=-a \lambda(1+\alpha \theta)$, it is well known that $k^{f}(\gamma)$ exists on $[0, \infty)$. Moreover, for $\lambda=\pi^{2} /(2 a \alpha)$ and $f(\theta)=-\pi^{2}(1+\alpha \theta) /(2 \alpha)$, it is well known that

(D) $k^{f}(0)=0$,

(E) $\lim _{\gamma \rightarrow \infty} k^{f}(\gamma)=(\pi / 2)(a \lambda \alpha)^{-1 / 2}=\sqrt{2} / 2>1 / 2$,

(F) $k^{f}(\gamma)$ is a strictly increasing function on $(0, \infty)$.

Hence, for $\lambda=\pi^{2} /(2 a \alpha)$, there exists a fixed number $\theta_{7}>0$ satisfying

$$
k^{f}\left(\gamma=\theta_{7}\right)=\frac{1}{2} \text {, }
$$

and

$$
k^{f}(\gamma)>\frac{1}{2} \quad \text { for } \quad \gamma>\theta_{7} .
$$

So by (4.4), for $0<\lambda<\pi^{2} /(2 a \alpha)$ and $\lambda$ small enough,

$$
\theta_{5}>\theta_{7},
$$

and hence it follows that

$$
k^{g}(\gamma)>k^{f}(\gamma)>\frac{1}{2} \text { for } \gamma>\theta_{5}\left(>\theta_{7}\right) .
$$

Thus, by above, problem (1.8) has a unique solution if $0<\lambda<\lambda_{2}$ and $\lambda$ is small enough.

(ii) For $\lambda \geq \lambda_{2}+\left(\pi^{2} /(a \alpha)\right)$,

$$
\begin{aligned}
g(\theta) & =\sigma q(\theta)-a \lambda(1+\alpha \theta) \\
& \leq\left[\sigma q(\theta)-a \lambda_{2}(1+\alpha \theta)\right]-\left(\pi^{2} / \alpha\right)(1+\alpha \theta) \\
& <-\pi^{2} \theta:=j(\theta) \quad \text { on }(0, \infty) .
\end{aligned}
$$


So by Lemma 2.3, we obtain that

$$
0<k^{g}(\gamma)<k^{j}(\gamma)=\frac{1}{2} \text { for all } \gamma \in(0, \infty) .
$$

Hence problem (1.8) has no solution for $\lambda \geq \lambda_{2}+\left(\pi^{2} /(a \alpha)\right)$. This completes the proof of Lemma 4.4 .

By Lemmas 2.1-2.3 and 4.1-4.4, for $g_{\lambda}(\theta)=\sigma q(\theta)-a \lambda(1+\alpha \theta)$, there exist two positive numbers $\underline{\lambda}<\bar{\lambda}$ with

$$
\underline{\lambda}=\frac{\pi^{2}}{a \alpha} \quad \text { and } \quad \lambda_{2}<\bar{\lambda}<\lambda_{2}+\frac{\pi^{2}}{a \alpha}
$$

satisfying

$$
\begin{gathered}
\lim _{\gamma \rightarrow \infty} k_{\underline{\lambda}}^{g}(\gamma)=(\pi / 2)(a \underline{\lambda} \alpha)^{-1 / 2}=\frac{1}{2}, \\
\max _{\gamma \in(0, \infty)} k_{\bar{\lambda}}^{g}(\gamma)=\frac{1}{2},
\end{gathered}
$$

such that Theorem 1.4 follows.

4.2. Proof of Theorem 1.5. We first recall from (1.17)-(1.19) that

$$
\begin{gathered}
16<\lambda_{0}<25, \\
25<\lambda_{1^{*}}<36, \\
140<\lambda_{2}<180 .
\end{gathered}
$$

(i) $(0 \leq \lambda \leq 25)$ For $\lambda=0$, problem (1.8) has a unique solution $\theta \equiv 0$. For $0<\lambda \leq 25$ $\left(<\lambda_{2}\right)$, by Lemma 4.2 , it is known that there exists a unique solution $\theta$ of problem (1.8) satisfying $0<\|\theta\|_{\infty}<\theta_{1}$. Moreover, for $q(\theta)$ given in Fig. 2, it is clear to see that

(A) For $0<\lambda<\lambda_{0}, g(\theta)=0$ has two positive roots $\theta_{1}<\theta_{4}\left(<\theta_{\infty} \approx 2200\right)$, with

$$
\int_{\theta_{1}}^{\theta_{\infty}} g(\theta) d \theta>0
$$

(B) For $\lambda=\lambda_{0}, g(\theta)=0$ has three positive roots $\theta_{1}<\theta_{2}<\theta_{4}\left(<\theta_{\infty}\right)$, with

$$
\int_{\theta_{1}}^{\theta_{\infty}} g(\theta) d \theta>0 .
$$

(C) For $\lambda_{0}<\lambda \leq 25\left(<\lambda_{1^{*}}\right), g(\theta)=0$ has four positive roots $\theta_{1}<\theta_{2}<\theta_{3}<\theta_{4}$ $\left(<\theta_{\infty}\right)$, with

$$
\int_{\theta_{1}}^{\theta_{3}} g(\theta) d \theta>0, \quad \int_{\theta_{3}}^{\theta_{\infty}} g(\theta) d \theta>0 .
$$

So in either case of (A)-(C), there exists no solution $\theta$ of problem (1.8) satisfying $\theta_{1} \leq\|\theta\|_{\infty}<\theta_{\infty}$. So problem (1.8) has a unique solution satisfying (1.20) for $0 \leq \lambda \leq 25$.

(ii) $\left(40 \leq \lambda \leq 140\left(<\lambda_{2}\right)\right)$ For $40 \leq \lambda \leq 140<\lambda_{2}, g(\theta)=0$ has exactly two positive roots $\theta_{1}<\theta_{2}\left(<\theta_{\infty}\right)$. Moreover,

$$
\int_{\theta_{1}}^{\theta_{\infty}} g(\theta) d \theta<0
$$


see Fig. 2. So there exists a number $\theta_{5}$ with $\theta_{2}<\theta_{5}<\theta_{\infty}$ satisfying $\int_{\theta_{1}}^{\theta_{5}} g(\theta) d \theta=0$ such that $k(\gamma)$ is defined for $\gamma \in\left[0, \theta_{1}\right) \cup\left(\theta_{5}, \theta_{\infty}\right)$. To show that problem (1.8) has at least two solutions satisfying (1.20) for $40 \leq \lambda \leq 140$, by Lemmas 2.1-2.3 and 4.1-4.3, it suffices to show that for $\lambda=40$ and $g_{40}(\theta)=\sigma q(\theta)-40 a(1+\alpha \theta)$,

$$
\inf _{\gamma \in\left(\theta_{5}, \theta_{\infty}\right)} k_{40}^{g}(\gamma)<\frac{1}{2}
$$

where

$$
k_{40}^{g}(\gamma):=\int_{0}^{\gamma} \frac{d u}{\left\{2\left[G_{40}(u)-G_{40}(\gamma)\right]\right\}^{1 / 2}} \quad\left(G_{40}(\theta)=\int_{0}^{\theta} g_{40}(u) d u\right) .
$$

Similarly as before, we show (4.6) by Lemma 2.3. For $\lambda=40$, assume that there exist numbers $n, s, t, m$, and $p$ satisfying

$$
n<0, \quad t>0, \quad p<0, \quad \theta_{1}<s<\theta_{2}<m<\theta_{\infty}
$$

such that the curve $\Lambda: f(\theta)=0$ which consists of two line segments connecting points $(0, n),(s, t)$, and $(m, p)$ point by point is below the curve $g_{40}(\theta)=0$ on $[0, m]$, i.e.,

$$
g_{40}(\theta)=\sigma q(\theta)-40 a(1+\alpha \theta)<f(\theta):= \begin{cases}\frac{(t-n) \theta}{s}+n & \text { for } 0 \leq \theta<s, \\ \frac{(p-t)(\theta-s)}{(m-s)}+t & \text { for } s \leq \theta \leq m,\end{cases}
$$

and $f$ satisfies

$$
\int_{r_{1}}^{m} f(\theta) d \theta<0
$$

where $r_{1}=n s /(n-t)$ is the solution of $f(\theta)=0$ on $(0, s)$. Let $r_{2}=(p s-m t) /(p-t)+s$ be the solution of $f(\theta)=0$ on $(s, m)$. By (4.9), there exists a number $r \in\left(r_{2}, m\right)$ such that

$$
\int_{r_{1}}^{r} f(\theta) d \theta=0
$$

see Fig. 7.

For $\lambda=40$, let

$$
k^{f}(\gamma):=\int_{0}^{\gamma} \frac{d u}{\{2[F(u)-F(\gamma)]\}^{1 / 2}} \quad\left(F(\theta)=\int_{0}^{\theta} f(u) d u\right) .
$$

It is clear that, in particular, $k_{40}^{g}(\gamma)$ and $k^{f}(\gamma)$ exist on $\left(\theta_{5}, \theta_{\infty}\right)$ and $(r, m)$ respectively. (Note that $\theta_{5}<r<m<\theta_{\infty}$.) By (4.8) and Lemma 2.3,

$$
0<k_{40}^{g}(\gamma)<k^{f}(\gamma) \text { for } \gamma \in(r, m)
$$

$k^{f}(\gamma)$ can be calculated explicitly since $f$ is piecewisely a line segment. Now for function $q(\theta)$ given in Fig. 2 and constants $\alpha=0.0039, \sigma=172.7, a=1286$, we choose

$$
n=-45000, \quad s=45, \quad t=190000, \quad m=2100, \quad p=-204000 .
$$

Then $r_{1}=405 / 47 \approx 8.6170, r_{2}=204090 / 197 \approx 1035.99, r \approx 2045.01$ and $(4.7)-(4.9)$ hold. Moreover, it can be computed that

$$
\inf _{\gamma \in(r, m)} k^{f}(\gamma)<0.22<\frac{1}{2}
$$




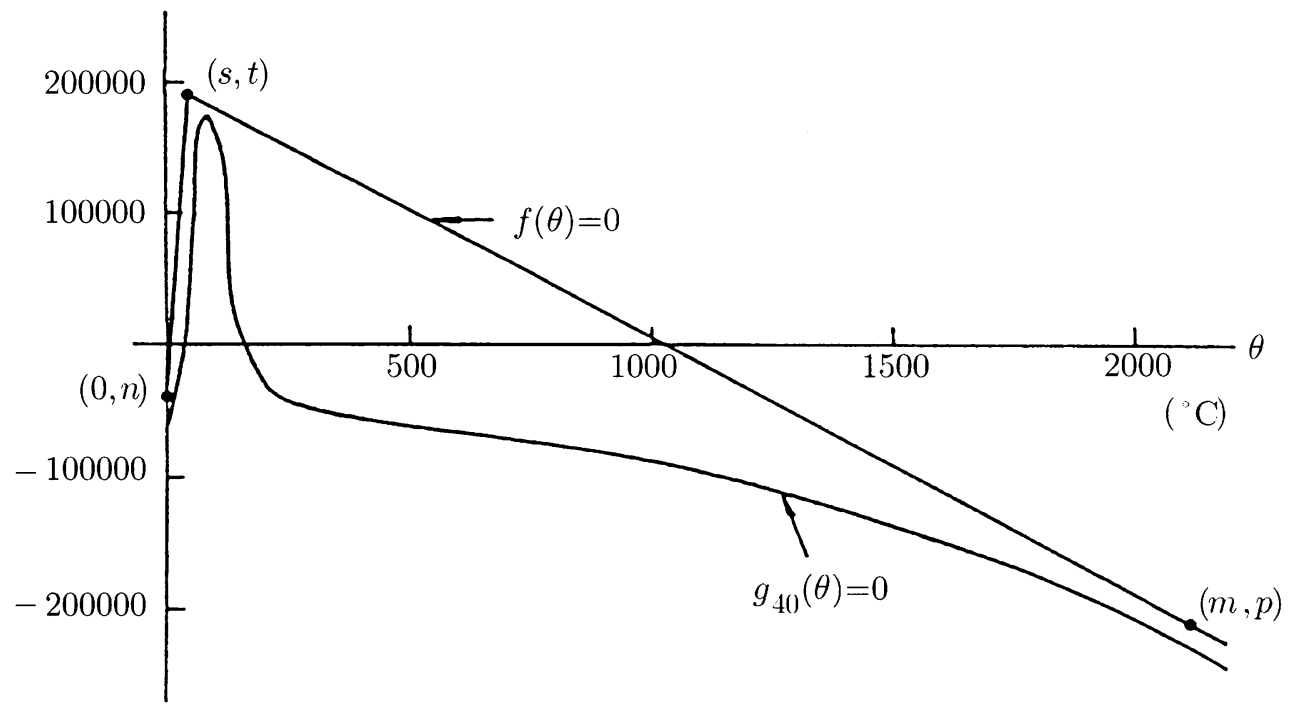

FIG. 7. Curves $f(\theta)=0$ and $g_{40}(\theta)=0$

see Fig. 8 which is obtained using the symbolic manipulator Mathematica. Thus (4.6) holds. It follows that problem (1.8) has at least two solutions satisfying (1.20) for $40 \leq$ $\lambda \leq 140$.

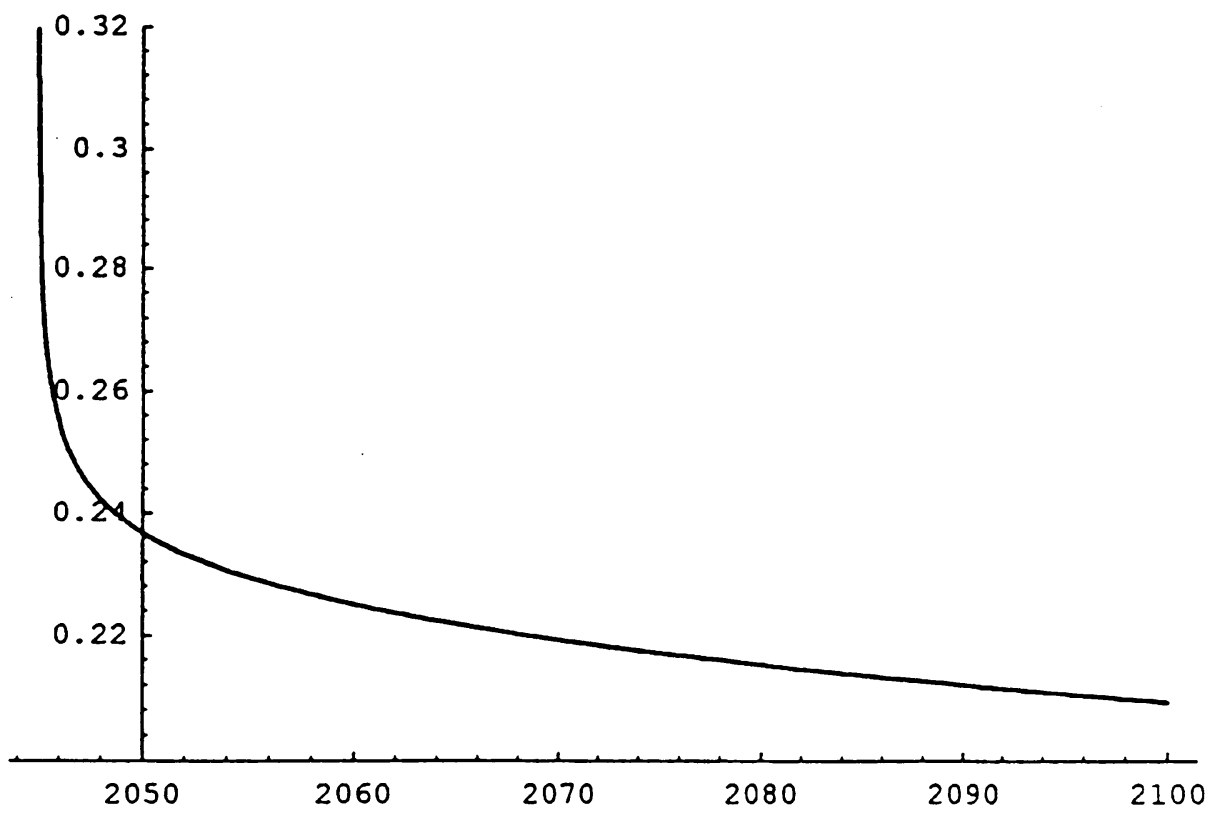

FIG. 8. Curves $k^{f}(\gamma)$ on $(r, m)$ 
(iii) $\left(\lambda \geq 182\left(>\lambda_{2}+\left(\pi^{2} /(a \alpha)\right)\right)\right)$ It follows from Lemma 4.4 and (1.19) that problem (1.8) has no solution satisfying (1.20) for

$$
\lambda \geq 182>180+\frac{\pi^{2}}{1286 \cdot 0.0039}>\lambda_{2}+\frac{\pi^{2}}{a \alpha} .
$$

(Note that $\pi^{2} /(1286 \cdot 0.0039) \approx 1.9679$.) This completes the proof of Theorem 1.5 .

Acknowledgment. The author thanks Prof. Stuart P. Hastings of the University of Pittsburgh and Prof. Maw-Ling Wang of the National Tsing Hua University (Taiwan) for valuable comments and discussions.

\section{REFERENCES}

[1] P. J. Berenson, Experiments on pool-boiling heat-transfer, Internat. J. Heat Mass Transfer 5, 985-999 (1962)

[2] A. E. Bergles, Poolboiling, Two-Phase Flows and Heat Transfer in the Power and Process Industries, Hemisphere Publishing Corporation, Chapter 7, 1981, pp. 191-225

[3] L. A. Bromley, Heat transfer in stable film boiling, Chem. Engrg. Progress 46, 221-227 (1950)

[4] R. C. Buck, Advanced Calculus, third edition, McGraw-Hill, New York, 1978, p. 118

[5] J. G. Collier, Convective Boiling and Condensation, second edition, McGraw-Hill, New York, 1981, pp. 121-133

[6] E. J. Doedel, G. Joly, and J. P. Kernevez, Continuation of a steep temperature front in nonlinear heat transfer, Semigroups, Theory and Applications, Vol. I, Pitman Res. Notes Math. Ser. 141, 96-109 (1984)

[7] G. Joly, J. P. Kernevez, and M. Llory, Thermal instability in pool boiling on wires at constant pressure, SIAM. J. Appl. Math. 43, 1294-1309 (1983)

[8] G. Joly, Analyse des solutions multiples dans les systèmes distribués, thèse, Compiènge, 1982

[9] T. Laetsch, The number of solutions of a nonlinear two-point boundary value problem, Indiana Univ. Math. J. 20, 1-13 (1970)

[10] N. Madsen, A graphical method for analyzing pool-boiling systems, Internat. J. Heat Mass Transfer 15, 513-517 (1973)

[11] S. Nukiyama, The maximum and minimum value of the heat $Q$ transmitted from metal to boiling water under atmospheric pressure, Journal Japan Society of Mechanical Engineers 37, 367-374 (1934). Translation in International Journal of Heat and Mass Transfer 9, 1419-1433 (1966)

[12] R. Schaaf, Global solution branches of two point boundary value problems, Lecture Notes in Math. 1458, Springer-Verlag, Berlin, Heidelberg, 1990

[13] S.-H. Wang and N. D. Kazarinoff, Bifurcation of steady-state solutions of a scalar reaction-diffusion equation in one space variable, J. Austral. Math. Soc. 52A, 343-355 (1992)

[14] A. Watson, Influence of axial wall conditions in variable property convection, with particular references to subcritical pressure fluids, Internat. J. Heat Mass Transfer 20, 65-71 (1967)

[15] T. Yanagida, Couple map lattice model for boiling, Physics Letter 165A, 405-408 (1992) 